\title{
DIFFERENTIAL GERSTENHABER ALGEBRAS ASSOCIATED TO NILPOTENT ALGEBRAS*
}

\author{
RICHARD CLEYTON ${ }^{\dagger}$ AND YAT-SUN POON ${ }^{\ddagger}$
}

\begin{abstract}
This article provides a complete description of the differential Gerstenhaber algebras of all nilpotent complex structures on any real six-dimensional nilpotent algebra. As an application, we classify all pseudo-Kählerian complex structures on six-dimensional nilpotent algebras whose differential Gerstenhaber algebra is quasi-isomorphic to that of the symplectic structure. In a weak sense of mirror symmetry, this gives a classification of pseudo-Kähler structures on six-dimensional nilpotent algebras whose mirror images are themselves.
\end{abstract}

Key words. Nilpotent algebra, Gerstenhaber algebra, complex structure, symplectic structure, deformation, mirror symmetry

AMS subject classifications. Primary 32G05; Secondary 32G07, 13D10, 16E45, 17B30, $53 \mathrm{D} 45$

1. Introduction. Nilmanifolds, i.e. compact quotients of simply connected nilpotent Lie groups, are known to be a rich source of exotic geometry. We are particularly interested in pseudo-Kähler geometry and its deformation theory on these spaces. We initially focus on the complex structures, and will bring symplectic structures in the picture at the end.

It is a general principle that the deformation theories of complex and symplectic structures are dictated by their associated differential Gerstenhaber algebras [8] [11] [18]. The associated cohomology theories are Dolbeault's cohomology with coefficients in the holomorphic tangent bundle, and de Rham's cohomology respectively. De Rham cohomology of nilmanifolds is known to be given by invariant differential forms [14] and there are several results for Dolbeault cohomology on nilmanifolds pointing in the same direction [2] [3]. Therefore, in this paper we focus on invariant objects, i.e. invariant complex structures and invariant symplectic forms on nilpotent Lie algebras.

Analysis and classification of invariant complex structures and pseudo-Kähler pairs on six-dimensional nilpotent algebras have been in progress in the past ten years [1] [2] [4] [16] [17]. In particular, it is known that a complex structure can be part of a pseudo-Kähler pair, only if it is nilpotent [6].

After a preliminary presentation on construction of differential Gerstenhaber algebra for invariant complex and symplectic structures, we give two key technical results, Proposition 10 and Proposition 11, describing the restrictive nature of quasiisomorphisms in our setting. We recall the definition of nilpotent complex structure in Section 3. Numerical invariants for these complex structures are identified, and used to refine older classifications. This in particular allows to identify the real algebra underlying a set of complex structure equations by evaluation of the invariants. The results of Section 3 including the invariants of complex structure equations and the associated underlying real algebras are summarized in Table 3.1.

In Section 4, we analyze the differential Gerstenhaber algebra DGA $(\mathfrak{g}, J)$ when a nilpotent complex structure $J$ on a nilpotent Lie algebra $\mathfrak{g}$ is given. The invariants

\footnotetext{
*Received November 2, 2007; accepted for publication March 19, 2008.

$\dagger$ Institute für Mathematik, Humboldt Universität zu Berlin, Unter den Linden 6, 10099 Berlin, Germany (cleyton@mathematik.hu-berlin.de).

${ }^{\ddagger}$ Department of Mathematics, University of California at Riverside, Riverside CA 92521, USA (ypoon@math.ucr.edu).
} 
of the complex structure equations dictate the structure of DGA $(\mathfrak{g}, J)$. Relying on the classification provided in Section 3 and Table 3.1, and in terms of the same set of invariants, we establish a relation between the Lie algebra structure of $\mathfrak{g}$ and that of $\operatorname{DGA}(\mathfrak{g}, J)$. The total output of Section 4 is provided in Theorem 24 and Table 4.1. These results demonstrate the phenomenon of "jumping" of DGA $(\mathfrak{g}, J)$ as $J$ varies through a family of nilpotent complex structures on some fixed algebra $\mathfrak{g}$. Results are given in Theorem 25 and Table 4.2. With the aid of Proposition 11, Theorem 24 we also show that each differential Gerstenhaber algebra DGA $(\mathfrak{g}, J)$ is isomorphic to a differential Gerstenhaber algebra DGA $(\mathfrak{h}, O)$ derived from a complex Lie algebra $\mathfrak{h}$ and linear isomorphism $O: \mathfrak{h} \rightarrow \mathfrak{h}^{*}$. The result is stated in Theorem 26. However, the map $O$ is not necessarily induced by a contraction with any symplectic form. A priori, it may not even be skew-symmetric.

Finally in Section 5 , we consider the differential Gerstenhaber algebra DGA $(\mathfrak{h}, \Omega)$ associated to an invariant symplectic structure $\Omega$ on a real nilpotent algebra $\mathfrak{h}$. We shall explain in Section 2 that $\operatorname{DGA}(\mathfrak{h}, \Omega)$ is essentially generated by the Lie algebra structure on $\mathfrak{h}$. This elementary observation, along with the results established in Section 4 and Table 4.1, allows us to answer the following question: Which sixdimensional nilpotent algebra $\mathfrak{g}$ admits a pseudo-Kähler structure $(J, \Omega)$ such that there is a quasi-isomorphism

$$
\operatorname{DGA}(\mathfrak{g}, J) \longrightarrow \operatorname{DGA}(\mathfrak{g}, \Omega) ?
$$

The construction of DGAs for complex structures and symplectic structures is well known (e.g. [18]). It is a key ingredient in homological mirror symmetry. Extending the concept of mirror symmetry, Merkulov considers the notion of weak mirror symmetry [11] [12]. In this paper, we call a Lie algebra $\mathfrak{g}$ with a complex structure $J$ and a Lie algebra $\mathfrak{h}$ with a symplectic structure $\Omega$ a "weak mirror pair" if there is a quasi-isomorphism between $\operatorname{DGA}(\mathfrak{g}, J)$ and $\operatorname{DGA}(\mathfrak{h}, \Omega)$. The aforementioned question stems from a consideration on when "self mirror" occurs. For four-dimensional nilpotent algebras, the answer could be derived from results in [15]. For six-dimensional nilpotent algebras, our answer is in Theorem 29.

\section{Differential Gerstenhaber Algebras.}

\subsection{Preliminaries.}

Definition 1. [7] [9, Definition 7.5.1] Let $R$ be a ring with unit and let $C$ be an $R$-algebra. Let $\mathfrak{a}=\oplus_{n \in \mathbb{Z}} \mathfrak{a}^{n}$ be a graded algebra over $C$. $\mathfrak{a}$ is a Gerstenhaber algebra if there is an associative product $\wedge$ and a graded commutative product $[-\bullet-]$ satisfying the following axioms. When $a \in \mathfrak{a}^{n}$, let $|a|$ denote its degree $n$. For $a \in \mathfrak{a}^{|a|}, b \in \mathfrak{a}^{|b|}$, $c \in \mathfrak{a}^{|c|}$,

$$
\begin{gathered}
a \wedge b \in \mathfrak{a}^{|a|+|b|}, b \wedge a=(-1)^{|a||b|} a \wedge b . \\
{[a \bullet b] \in \mathfrak{a}^{|a|+|b|-1},[a \bullet b]=-(-1)^{(|a|+1)(|b|+1)}[b \bullet a] .} \\
(-1)^{(|a|+1)(|c|+1)}[[a \bullet b] \bullet c]+(-1)^{(|b|+1)(|a|+1)}[[b \bullet c] \bullet a]+(-1)^{(|c|+1)(|b|+1)}[[c \bullet a] \bullet b]=0 \\
{[a \bullet b \wedge c]=[a \bullet b] \wedge c+(-1)^{(|a|+1)|b|} b \wedge[a \bullet c] .}
\end{gathered}
$$

On the other hand, we have the following construction. 
Definition 2. A differential graded algebra is a graded algebra $\mathfrak{a}=\oplus_{n \in \mathbb{Z}} \mathfrak{a}^{n}$ with a graded commutative product $\wedge$ and a differential d of degree +1 , i.e. a map $d: \mathfrak{a} \rightarrow \mathfrak{a}$ such that

$$
d\left(\mathfrak{a}^{n}\right) \subseteq \mathfrak{a}^{n+1}, \quad d \circ d=0, \quad d(a \wedge b)=d a \wedge b+(-1)^{|a|} a \wedge d b .
$$

Definition 3. Let $\mathfrak{a}=\oplus_{n \in \mathbb{Z}} \mathfrak{a}^{n}$ be a graded algebra over $C$ such that $(\mathfrak{a},[-\bullet-], \wedge)$ form a Gerstenhaber algebra and $(\mathfrak{a}, \wedge, d)$ form a differential graded algebra. If in addition

$$
d[a \bullet b]=[d a \bullet b]+(-1)^{|a|+1}[a \bullet d b],
$$

for all $a$ and $b$ in $\mathfrak{a}$, then $(\mathfrak{a},[-\bullet-], \wedge, d)$ is a differential Gerstenhaber algebra (DGA).

For any Gerstenhaber algebra, $\mathfrak{a}^{1}$ with the induced bracket is a Lie algebra. Conversely, suppose $\mathfrak{a}^{1}$ is a finite dimensional algebra over the complex or real numbers, equipped with a differential compatible with the Lie bracket. Then a straightforward induction allows one to construct a DGA structure on the exterior algebra of $\mathfrak{a}^{1}$.

Lemma 4. Let $\mathfrak{a}^{1}$ be a finite dimensional Lie algebra with bracket $[-\bullet-]$. Let $\mathfrak{a}$ be the exterior algebra generated by $\mathfrak{a}^{1}$. Then the Lie bracket on $\mathfrak{a}^{1}$ uniquely extends to a bracket on $\mathfrak{a}$ so that $(\mathfrak{a},[-\bullet-], \wedge)$ is a Gerstenhaber algebra.

If, furthermore, an operator $d: \mathfrak{a}^{1} \rightarrow \mathfrak{a}^{2}$ is extended as in (5), then $(\mathfrak{a},[-\bullet-], \wedge)$ is a differential Gerstenhaber algebra if and only if

$$
d[a \bullet b]=[d a \bullet b]+[a \bullet d b]
$$

for all $a$ and $b$ in $\mathfrak{a}^{1}$.

Definition 5. A homomorphism of differential graded Lie algebras is called a quasi-isomorphism if the map induced on the associated cohomology groups is a linear isomorphism.

A quasi-isomorphism of differential Gerstenhaber algebras is a homomorphism of $D G A$ s that descends to an isomorphism of cohomology groups.

Note that in the latter case the isomorphism is one of Gerstenhaber algebras.

2.2. DGA of complex structures. Suppose $J$ is an integrable complex structure on $\mathfrak{g}$. i.e. $J$ is an endomorphism of $\mathfrak{g}$ such that $J \circ J=-1$ and

$$
[x \bullet y]+J[J x \bullet y]+J[x \bullet J y]-[J x \bullet J y]=0 .
$$

Then the $\pm i$ eigenspaces $\mathfrak{g}^{(1,0)}$ and $\mathfrak{g}^{(0,1)}$ are complex Lie subalgebras of the complexified algebra $\mathfrak{g}_{\mathbb{C}}$. Let $\mathfrak{f}$ be the exterior algebra generated by $\mathfrak{g}^{(1,0)} \oplus \mathfrak{g}^{*(0,1)}$, i.e.

$$
\mathfrak{f}^{n}:=\wedge^{n}\left(\mathfrak{g}^{(1,0)} \oplus \mathfrak{g}^{*(0,1)}\right), \quad \text { and } \quad \mathfrak{f}=\oplus_{n} \mathfrak{f}^{n} .
$$

The integrability condition in (8) implies that $\mathfrak{f}^{1}$ is closed under the Courant bracket

$$
[x+\alpha \bullet y+\beta]:=[x, y]+\iota_{x} d \beta-\iota_{y} d \alpha .
$$

A similar construction holds for the conjugate $\overline{\mathfrak{f}}$, generated by $\mathfrak{g}^{(0,1)} \oplus \mathfrak{g}^{*(1,0)}$. 
Recall that if $(\mathfrak{g},[-\bullet-])$ is a Lie algebra, the Chevalley-Eilenberg (C-E) differential $d$ is defined on the dual vector space $\mathfrak{g}^{*}$ by the relation

$$
d \alpha(x, y):=-\alpha([x \bullet y]),
$$

for $\alpha \in \mathfrak{g}^{*}$ and $x, y \in \mathfrak{g}$. This operator is extended to the exterior algebra $\wedge \mathfrak{g}^{*}$ by derivation. The identity $d \circ d=0$ is equivalent to the Jacobi identity for the Lie bracket $[-\bullet-]$ on $\mathfrak{g}$. It follows that $\left(\wedge \mathfrak{g}^{*}, d\right)$ is a differential graded algebra.

The natural pairing on $\left(\mathfrak{g} \oplus \mathfrak{g}^{*}\right) \otimes \mathbb{C}$, induces a complex linear isomorphism $\left(\mathfrak{f}^{1}\right)^{*} \cong$ $\overline{\mathfrak{f}}^{-1}$. Therefore, the C-E differential of the Lie algebra $\bar{f}^{-1}$ is a map from $\mathfrak{f}^{1}$ to $\mathfrak{f}^{2}$. Denote this operator by $\bar{\partial}$. Similarly, we denote the C-E differential of $\mathfrak{f}^{1}$ by $\partial$. It is well known that the maps

$$
\bar{\partial}: \mathfrak{g}^{*(0,1)} \rightarrow \wedge^{2} \mathfrak{g}^{*(0,1)}, \quad \text { and } \quad \bar{\partial}: \mathfrak{g}^{(1,0)} \rightarrow \mathfrak{g}^{(1,0)} \otimes \mathfrak{g}^{*(0,1)}
$$

are respectively given by

$$
\bar{\partial} \bar{\omega}=(d \bar{\omega})^{0,2}, \quad(\bar{\partial} T) \bar{W}=[\bar{W} \bullet T]^{1,0}
$$

for any $\omega$ in $\mathfrak{g}^{*(0,1)}, T \in \mathfrak{g}^{1,0}$ and $\bar{W} \in \mathfrak{g}^{0,1}$.

If $\left\{T_{\ell}: 1 \leq \ell \leq n\right\}$ forms a basis for $\mathfrak{g}^{1,0}$ and $\left\{\omega^{\ell}: 1 \leq \ell \leq n\right\}$ the dual basis in $\mathfrak{g}^{*(1,0)}$, then we have

$$
\bar{\partial} \bar{\omega}^{\ell}=\left(d \bar{\omega}^{\ell}\right)^{0,2}, \quad(\bar{\partial} T)=\sum_{\ell} \bar{\omega}^{\ell} \wedge\left[\bar{T}_{\ell} \bullet T_{j}\right]^{1,0} .
$$

Based on Lemma 4, it is an elementary computation to verify that the quadruples $(\mathfrak{f},[-\bullet-], \wedge, \bar{\partial})$ and $(\overline{\mathfrak{f}},[-\bullet-], \wedge, \partial)$ are differential Gerstenhaber algebras.

For a given Lie algebra $\mathfrak{g}$ and a choice of invariant complex structure $J$, we denote the differential Gerstenhaber algebra $(\mathfrak{f},[-\bullet-], \wedge, \bar{\partial})$ by DGA $(\mathfrak{g}, J)$.

The following observation relying on the nature of the $\bar{\partial}$ and $\partial$ will be helpful, although apparently obvious.

Lemma 6. Given a complex linear identification $\overline{\mathfrak{f}}^{1} \cong\left(\mathfrak{f}^{1}\right)^{*}$, the Lie algebras $\left(\mathfrak{f}^{1},[-\bullet-]\right),\left(\overline{\mathfrak{f}}^{1},[-\bullet-]\right)$ and the graded differential algebras $(\mathfrak{f}, \bar{\partial}),(\overline{\mathfrak{f}}, \partial)$ determine each other.

2.3. DGA of symplectic structures. Let $\mathfrak{h}$ be a Lie algebra over $\mathbb{R}$. The exterior algebra of the dual $\mathfrak{h}^{*}$ with the C-E differential $d$ is a differential graded Lie algebra.

Suppose that $O: \mathfrak{h} \rightarrow \mathfrak{h}^{*}$ is a real linear map. Define a bracket $[-\bullet-]_{O}$ on $\mathfrak{h}^{*}$ by

$$
[\alpha \bullet \beta]_{O}:=O\left[O^{-1} \alpha \bullet O^{-1} \beta\right] .
$$

It is a tautology that $\left(\mathfrak{h}^{*},[-\bullet-]_{O}\right)$ becomes a Lie algebra, with the map $O$ understood as a Lie algebra homomorphism.

Definition 7. A linear map $O: \mathfrak{h} \rightarrow \mathfrak{h}^{*}$ from a Lie algebra to its dual is said to be compatible with the $C$-E differential if for any $\alpha, \beta$ in $\mathfrak{h}^{*}$,

$$
d[\alpha \bullet \beta]_{O}=[d \alpha \bullet \beta]_{O}+[\alpha \bullet d \beta]_{O} .
$$

Due to Lemma 4, the next observation is a matter of definitions. 
Lemma 8. Suppose $\mathfrak{h}$ is a Lie algebra, and take an element $O$ in $\operatorname{Hom}\left(\mathfrak{h}, \mathfrak{h}^{*}\right)$ compatible with the $C$-E differential. Then $\left(\wedge \bullet \mathfrak{h}^{*},[-\bullet-]_{O}, \wedge, d\right)$ is a differential Gerstenhaber algebra.

When the algebra $\mathfrak{h}$ has a symplectic form $\Omega$, the contraction with $\Omega$ defines an $O$ as in the above lemma. In this case, the differential Gerstenhaber algebra $\left(\wedge^{\bullet} \mathfrak{h}^{*},[-\bullet-]_{\Omega}, \wedge, d\right)$ after complexification is denoted by DGA $(\mathfrak{h}, \Omega)$.

\section{Complex Structures on Nilpotent Algebras.}

3.1. General Theory. Let $\mathfrak{g}$ be a Lie algebra over $\mathbb{R}$ or $\mathbb{C}$. The lower central series of $\mathfrak{g}$ is the sequence of subalgebras $\mathfrak{g}_{p+1} \subset \mathfrak{g}_{p} \subset \mathfrak{g}$ given by

$$
\mathfrak{g}_{0}=\mathfrak{g}, \quad \mathfrak{g}_{p}=\left[\mathfrak{g}_{p-1} \bullet \mathfrak{g}\right] .
$$

A Lie algebra $\mathfrak{g}$ is $s$-step nilpotent if $s$ is the smallest integer such that $\mathfrak{g}_{s}=\{0\}$. Defining $V_{p}$ to be the annihilator of $\mathfrak{g}_{p}$ one has the dual sequence $\mathfrak{g}^{*} \supset V_{p} \supset V_{p+1}$. The dual sequence may also be defined recursively as

$$
V_{0}=\{0\}, \quad V_{p}=\left\{\alpha \in \mathfrak{g}^{*}: d \alpha \in \Lambda^{2} V_{p-1}\right\} .
$$

We note that if the subscript $\mathbb{C}$ denotes complexification of vector spaces and Lie algebras, then $V_{p}(\mathfrak{g})_{\mathbb{C}}=V_{p}\left(\mathfrak{g}_{\mathbb{C}}\right)$. Write $n_{p}=\operatorname{dim} V_{p}$. A Malcev basis for $\mathfrak{g}^{*}$ is a basis chosen such that $e^{1}, \ldots, e^{n_{1}}$ is a basis for $V_{1}$, supplemented with $e^{n_{1}+1}, \ldots, e^{n_{2}}$ to form a basis for $V_{2}$, et cetera. For such a basis one has $d e^{p} \in \Lambda^{2}\left\langle e^{1}, \ldots, e^{p-1}\right\rangle$. The short-hand notation $12:=e^{12}:=e^{1} \wedge e^{2}$ is convenient. Using this one may identify a Lie algebra by listing its structure equations with respect to a Malcev basis as $\left(d e^{1}, \ldots, d e^{n}\right)$. For instance we may write $\mathfrak{g}=(0,0, a 12)$ to mean the Lie algebra $\mathfrak{g}$ generated by the relations $d e^{1}=0=d e^{2}, d e^{3}=a e^{1} \wedge e^{2}$. This has the single non-trivial bracket $\left[e_{1} \bullet e_{2}\right]=-a e_{3}$.

Lemma 9. Suppose $\mathfrak{h}$ and $\mathfrak{k}$ are Lie algebras of the same dimension, $\mathfrak{h}$ is nilpotent and $\phi:\left(\wedge \mathfrak{h}^{*}, d\right) \rightarrow\left(\wedge \mathfrak{k}^{*}, d\right)$ is a quasi-isomorphism of the associated differential graded algebras. Then $\phi$ is an isomorphism.

Proof. If $\phi: \mathfrak{g}^{*} \rightarrow \mathfrak{h}^{*}$ is a homomorphism of the associated differential graded algebras then $\phi\left(V_{p}(\mathfrak{g})\right) \subset V_{p}(\mathfrak{h})$. When $\phi$ is furthermore a quasi-isomorphism then the restriction $V_{1}(\mathfrak{g}) \rightarrow V_{1}(\mathfrak{h})$ is an isomorphism of vector spaces. Suppose that $\phi$ restricted to $V_{p-1}(\mathfrak{g})$ is an isomorphism onto $V_{p-1}(\mathfrak{h})$. Then clearly the induced map $\Lambda^{2} V_{p-1}(\mathfrak{g}) \rightarrow \Lambda^{2} V_{p-1}(\mathfrak{h})$ is also an isomorphism. Suppose that $a \in V_{p}(\mathfrak{g})$ satisfies $\phi(a)=0$. Then $d a \in \Lambda^{2} V_{p-1}(\mathfrak{g})$ satisfies $\phi(d a)=0$. But then $d a=0$, so $a \in V_{1}$, and $\phi(a)=0$ actually implies $a=0$.

Proposition 10. Suppose that $\mathfrak{g}$ and $\mathfrak{h}$ are finite dimensional nilpotent Lie algebras of the same dimension, $J$ is an integrable complex structure on $\mathfrak{g}$ and $O: \mathfrak{h} \rightarrow \mathfrak{h}^{*}$ is a linear map compatible with the $C$-E differential on $\mathfrak{h}$. Then a homomorphism $\phi$ from $\operatorname{DGA}(\mathfrak{g}, J)$ to $\operatorname{DGA}(\mathfrak{h}, O)$ is a quasi-isomorphism if and only if it is an isomorphism.

Proof. As a quasi-isomorphism of DGAs, $\phi$ is a quasi-isomorphism of the underlying exterior differential algebras:

$$
\phi:\left(\wedge^{*} \mathfrak{f}^{1}, \wedge, \bar{\partial}\right) \rightarrow\left(\wedge^{*} \mathfrak{h}_{\mathbb{C}}^{*}, \wedge, d\right) .
$$

The last lemma shows that it has to be an isomorphism. 
Two special types of complex DGAs were introduced above: Those coming from an integrable complex structure $J$ on a real algebra $\mathfrak{g}$, denoted DGA $(\mathfrak{g}, J)$ and those derived from a linear identification $O: \mathfrak{h} \rightarrow \mathfrak{h}^{*}$ compatible with the differential. For the latter we write $\operatorname{DGA}(\mathfrak{h}, O)$. A problem related to "weak mirror symmetry" is: given an algebra $\mathfrak{g}$ and a complex structure $J$, when does an $\mathfrak{h}$ and $O$ exist so that $\operatorname{DGA}(\mathfrak{g}, J)$ is quasi-isomorphic to $\operatorname{DGA}(\mathfrak{h}, O)$ ? For nilpotent algebras we shall see below that this always is the case.

Given $J$ on $\mathfrak{g}$ write $\mathfrak{k}$ for the Lie algebra $\overline{\mathfrak{f}}^{1}$ consisting of degree one elements in $\operatorname{DGA}(\mathfrak{g}, J)$. As $\mathfrak{h}$ is complex we may speak of the complex conjugate algebra consisting of the conjugate vector space $\overline{\mathfrak{h}}$. Let $c: \mathfrak{h} \rightarrow \overline{\mathfrak{h}}$ be the canonical map such that $c(a x)=\bar{a} x$ for complex $a$ and $x$ in $\mathfrak{h}$. Then $[x, y]_{c}:=c[c(x), c(y)]$ equips $\overline{\mathfrak{h}}$ with a Lie bracket. We say that $\mathfrak{h}$ is self-conjugate if a complex linear isomorphism $\mathfrak{h} \rightarrow \overline{\mathfrak{h}}$ exists.

Proposition 11. Let $\mathfrak{g}$ be a Lie algebra with complex structure $J$. Let DGA $(\mathfrak{g}, J)$ be its differential Gerstenhaber algebra. Write $\mathfrak{h}$ for the Lie algebra $\mathfrak{f}^{1}$ and suppose that $\mathfrak{h}$ is self-conjugate. Then there exists a complex linear isomorphism $O: \mathfrak{h} \rightarrow$ $\mathfrak{h}^{*}$ compatible with the $C$-E differential $d$ on $\mathfrak{h}$ so that $\mathrm{DGA}(\mathfrak{h}, O)$ is isomorphic to $\operatorname{DGA}(\mathfrak{g}, J)$.

Proof. We construct the map $O$. Let $\phi: \mathfrak{h} \rightarrow \mathfrak{f}^{1}$ be the identification of $\mathfrak{h}$ as the Lie algebra given by $\mathfrak{f}^{1}$. Composing on both sides with complex conjugation gives the isomorphism $\bar{\phi}:=c \circ \phi \circ c: \overline{\mathfrak{h}} \rightarrow \overline{\mathfrak{f}}^{1}$ of Lie algebras. Taking the identifications $\overline{\mathfrak{f}}^{1} \cong\left(\mathfrak{f}^{1}\right)^{*}$ and $\overline{\mathfrak{h}} \cong \mathfrak{h}$ into account gives the isomorphism

$$
\psi: \mathfrak{h} \cong \overline{\mathfrak{h}} \stackrel{\bar{\phi}}{\rightarrow} \overline{\mathfrak{f}}^{1} \cong\left(\mathfrak{f}^{1}\right)^{*}
$$

of Lie algebras. The dual map $\psi^{*}$ is now an isomorphism of exterior differential algebras

$$
\psi^{*}: \wedge^{*} \mathfrak{f}^{1} \rightarrow \wedge^{*} \mathfrak{h}^{*}, \quad \psi^{*} \circ \bar{\partial}=d \circ \psi^{*} .
$$

We claim that the following composition

$$
O: \mathfrak{h} \stackrel{\phi}{\rightarrow} \mathfrak{f}^{1} \stackrel{\psi^{*}}{\rightarrow} \mathfrak{h}^{*}
$$

is compatible with $d$. By Lemma 4 this is the case if equation (7) holds for the bracket $[\alpha \bullet \beta]_{O}=: O\left[O^{-1} \alpha \bullet O^{-1} \beta\right]$. But

$$
[\alpha \bullet \beta]_{O}=\psi^{*} \circ \phi\left[\phi^{-1} \circ\left(\psi^{*}\right)^{-1} \alpha \bullet \phi^{-1} \circ\left(\psi^{*}\right)^{-1} \beta\right]=\psi^{*}\left[\left(\psi^{*}\right)^{-1} \alpha \bullet\left(\psi^{*}\right)^{-1} \beta\right] .
$$

Hence,

$$
\begin{aligned}
d[\alpha \bullet \beta]_{O} & =d\left(\psi^{*}\left[\left(\psi^{*}\right)^{-1} \alpha \bullet\left(\psi^{*}\right)^{-1} \beta\right]\right) \\
& =\psi^{*}\left(\bar{\partial}\left[\left(\psi^{*}\right)^{-1} \alpha \bullet\left(\psi^{*}\right)^{-1} \beta\right]\right) \\
& \left.=\psi^{*}\left(\left[\bar{\partial}\left(\psi^{*}\right)^{-1} \alpha \bullet\left(\psi^{*}\right)^{-1} \beta\right]\right)+\psi^{*}\left(\left[\left(\psi^{*}\right)^{-1} \alpha \bullet \bar{\partial}\left(\psi^{*}\right)^{-1} \beta\right]\right)\right) \\
& \left.=\psi^{*}\left(\left[\left(\psi^{*}\right)^{-1} d \alpha \bullet\left(\psi^{*}\right)^{-1} \beta\right]\right)+\psi^{*}\left(\left[\left(\psi^{*}\right)^{-1} \alpha \bullet\left(\psi^{*}\right)^{-1} d \beta\right]\right)\right) \\
& =[d \alpha \bullet \beta]_{O}+[\alpha \bullet d \beta]_{O} .
\end{aligned}
$$

By Lemma $8, \operatorname{DGA}(\mathfrak{k}, O):=\left(\wedge^{*} \mathfrak{k}^{*},[-\bullet-]_{O}, \wedge, d\right)$ forms a differential Gerstenhaber algebra. It is clear from (18) and (20) that the map $\psi^{*}$ yields an isomorphism from $\operatorname{DGA}(\mathfrak{g}, J)$ to $\operatorname{DGA}(\mathfrak{k}, O)$. 
It should be noted that the map $O$ is not necessarily skew-symmetric, nor is it automatically closed when it is skew. In particular the DGA structure obtained above does not necessarily arise from contraction with a symplectic structure. Also note that the condition $\overline{\mathfrak{k}} \cong \mathfrak{k}$ is satisfied precisely when $\mathfrak{k}$ is the complexification of some real algebra. Whilst in the context of six-dimensional nilpotent algebras this is always the case, there exist non-isomorphic real algebras having the same complexification.

3.2. Nilpotent complex structures. An almost complex structure $J$ on $\mathfrak{g}$ may be given by a choice of basis $\omega=\left\{\omega^{k}, 1 \leq k \leq m\right\}, 2 m=\operatorname{dim}_{\mathbb{R}} \mathfrak{g}$, of the space of $(1,0)$-forms in the complexified dual $\mathfrak{g}_{\mathbb{C}}^{*}$. Such a basis may equivalently be given as a basis $e=\left(e^{1}, \ldots, e^{2 m}\right)$ of $\mathfrak{g}^{*}$ so that $e^{2}=J e^{1}$, or $\omega^{1}=e^{1}+i e^{2}$, and so on. When $e$ and $\omega$ are related in this way we will write $e=e(\omega)$ or $\omega=\omega(e)$. The almost complex structure is then integrable or simply a complex structure if the ideal in $\Lambda^{*} \mathfrak{g}_{\mathbb{C}}^{*}$ generated by the $(1,0)$-forms is closed under exterior differentiation. For a nilpotent Lie algebra, an almost complex structure is integrable if there exists a basis $\left(\omega^{j}\right)$ of $(1,0)$-forms so that $d \omega^{1}=0$ and for $j>1, d \omega^{j}$ lies in the ideal generated by $\omega^{1}, \ldots, \omega^{j-1}$. Equivalently,

$$
0=d\left(\omega^{1} \wedge \omega^{2} \wedge \cdots \wedge \omega^{p}\right), \quad p=1, \ldots, m .
$$

Let the set of such bases be denoted $\Omega(\mathfrak{g}, J)$.

On nilpotent Lie algebras certain complex structures are distinguished. Among these are complex structures such that $[X, J Y]=J[X, Y]$. Equivalently, $d \omega^{p} \in$ $\Lambda^{2}\left\langle\omega^{1}, \ldots, \omega^{p-1}\right\rangle$. These are the complex structures for which $\mathfrak{g}$ is the real algebra underlying a complex Lie algebra. At the opposite end to these are the abelian complex structures which satisfy $[J X, J Y]=[X, Y][1]$. Equivalently, the $+i$-eigenspace of $J$ in $\mathfrak{g}_{\mathbb{C}}$ is an abelian subalgebra of $\mathfrak{g}_{\mathbb{C}}$. In particular abelian $J_{\mathrm{S}}$ are always integrable. In terms of $(1,0)$-forms a complex structure is abelian if and only if there exists an $\omega$ in $\Omega(\mathfrak{g}, J)$ such that $d \omega^{j}$ is in the intersection of the two ideals generated by $\omega^{1}, \ldots, \omega^{j-1}$ and $\bar{\omega}^{1}, \ldots, \bar{\omega}^{j-1}$, respectively.

The concept of abelian complex structures may be generalized to that of nilpotent complex structures [4]. A nilpotent almost complex structure may be defined as an almost complex structure with a basis of $(1,0)$-forms such that

$$
d \omega^{p} \in \Lambda^{2}\left\langle\omega^{1}, \ldots, \omega^{p-1}, \bar{\omega}^{1}, \ldots, \bar{\omega}^{p-1}\right\rangle .
$$

For a given algebra $\mathfrak{g}$ and nilpotent almost complex structure $J$ we write $P(\mathfrak{g}, J)$ for the set of such bases. Nilpotent almost complex structures are not necessarily integrable. If a nilpotent $J$ is integrable, then $P(\mathfrak{g}, J) \subset \Omega(\mathfrak{g}, J)$. A nilpotent complex structure is abelian if and only if

$$
0=d\left(\bar{\omega}^{1} \wedge \bar{\omega}^{2} \wedge \cdots \wedge \bar{\omega}^{p-1} \wedge \omega^{p}\right), \quad p=1, \ldots, m .
$$

It is apparent that abelian complex structures are nilpotent.

In subsequent presentation, we suppress the wedge product sign.

3.3. Six-dimensional algebras. Some of the results of this section may be regarded as a re-organization of past results in terms of invariants relevant to our further analysis. Our key references are [16] and [17]. To name specific isomorphism classes of six-dimensional nilpotent Lie algebras, we use the notation $\mathfrak{h}_{n}$ as given in [4]. 
Suppose then $\operatorname{dim}_{\mathbb{R}} \mathfrak{g}=6$. Let $J$ be a nilpotent almost complex structure on $\mathfrak{g}$. The structure equations for an integrable element $\omega$ in $P(\mathfrak{g}, J)$ are [4]

$$
\left\{\begin{array}{l}
d \omega^{1}=0, \\
d \omega^{2}=\epsilon \omega^{1} \bar{\omega}^{1}, \\
d \omega^{3}=\rho \omega^{1} \omega^{2}+A \omega^{1} \bar{\omega}^{1}+B \omega^{1} \bar{\omega}^{2}+C \omega^{2} \bar{\omega}^{1}+D \omega^{2} \bar{\omega}^{2},
\end{array}\right.
$$

for complex numbers $\epsilon, \rho, A, B, C, D$. Note that $d d \omega^{3}=0$ forces $D \epsilon=0$. Moreover, if $\epsilon$ is not zero, $\omega^{3}$ may be replaced with $\epsilon \omega^{3}-A \omega^{2}$ so after re-scaling the $\omega^{j}$ one obtains the reduced structure equations [17]

$$
\left\{\begin{array}{l}
d \omega^{1}=0 \\
d \omega^{2}=\epsilon \omega^{1} \bar{\omega}^{1} \\
d \omega^{3}=\rho \omega^{1} \omega^{2}+(1-\epsilon) A \omega^{1} \bar{\omega}^{1}+B \omega^{1} \bar{\omega}^{2}+C \omega^{2} \bar{\omega}^{1}+(1-\epsilon) D \omega^{2} \bar{\omega}^{2}
\end{array}\right.
$$

where $\epsilon$ and $\rho$ are either 0 or 1 and $A, B, C, D$ are complex numbers.

To avoid ambiguity we rule out the case $\epsilon \neq 0, d \omega^{3}=0$ for any form of the structure equations as this is equivalent to $\epsilon=0, d \omega^{3}=\omega^{1} \bar{\omega}^{1}$.

Given structure equations (24) for a nilpotent complex structure, we will calculate $\operatorname{DGA}(\mathfrak{g}, J)$ in Section 4. However, if we take (24) as a starting point, it is not obvious to recognize the real algebra $\mathfrak{g}$ which underlies the complex structure. We shall first provide a way to do this that will fit the purpose of this paper.

For this task, we identify invariants of $P(\mathfrak{g}, J)$. The most immediate invariants are the dimensions of the vector spaces in the dual sequence $V_{0} \subset V_{1} \subset \ldots$ for $\mathfrak{g}_{\mathbb{C}}$. As the inclusions

$$
V_{1} \supset\left\langle\omega^{1}, \bar{\omega}^{1}, \omega^{2}+\bar{\omega}^{2}\right\rangle, \quad V_{2} \supset\left\langle\omega^{1}, \bar{\omega}^{1}, \omega^{2}, \bar{\omega}^{2}\right\rangle
$$

always hold, $V_{3}=\mathfrak{g}_{\mathbb{C}}^{*}$ for any 6-dimensional nilpotent algebra with nilpotent complex structure. Define

$$
n=\left(n_{1}, n_{2}\right)=\left(\operatorname{dim} V_{1}, \operatorname{dim} V_{2}\right) .
$$

We now collect several facts on these particular invariants.

LEMMA 12. Given a nilpotent complex structure $J$ on a six-dimensional nilpotent algebra $\mathfrak{g}$, the following hold:

(a) $3 \leq n_{1} \leq 6,4 \leq n_{2} \leq 6$ and $n_{1} \leq n_{2}$.

(b) There exists $\omega$ in $P(\mathfrak{g}, J)$ such that $\epsilon=0$ or $\epsilon=1$.

(c) If $\epsilon=1$, there exists $\omega$ in $P(\mathfrak{g}, J)$ such that $A=D=0$.

(d) If $\epsilon=0$, then $n_{2}=6$.

(e) $\rho=0$ if and only if $J$ is an abelian complex structure.

(f) Let $d$ be the dimension of the complex linear span of $d \omega^{3}$ and $d \bar{\omega}^{3}$. Then $d \leq 1$ if and only if

$$
\rho=0, \quad|B|^{2}=|C|^{2}, \quad A \bar{D}=\bar{A} D, \quad A \bar{B}=\bar{A} C, \quad D \bar{B}=\bar{D} C .
$$

Based on the above information, we re-organize some of the data from $[17$, Theorem 2.9] and [16, Table A.1].

LEMMA 13. Suppose a complex structure on $\mathfrak{g}$ is given with structure constants as in (24) with $\epsilon \in\{0,1\}$. 
(a) $n=(6,6)$ if and only if $\mathfrak{g} \cong \mathfrak{h}_{1}=(0,0,0,0,0,0)$.

(b) $n=(5,6)$ if and only if $\epsilon=0$ and $d=1$. In this case,

$$
\mathfrak{g} \cong\left\{\begin{array}{l}
\mathfrak{h}_{8}=(0,0,0,0,0,12), \\
\mathfrak{h}_{3}=(0,0,0,0,0,12+34) .
\end{array}\right.
$$

(c) If $n=(4,6)$, then $\epsilon=0$ and $d=2$. The Lie algebra is

$$
\mathfrak{g} \cong\left\{\begin{array}{l}
\mathfrak{h}_{6}=(0,0,0,0,12,13), \\
\mathfrak{h}_{2}=(0,0,0,0,12,34), \\
\mathfrak{h}_{4}=(0,0,0,0,12,13+42), \\
\mathfrak{h}_{5}=(0,0,0,0,13+42,14+23) .
\end{array}\right.
$$

(d) If $n=(3,6)$, then $\epsilon=1, \rho \neq 0$, and there exists an element $\sigma$ in $P(\mathfrak{g}, J)$ such that $d \sigma^{3}=\sigma^{1}\left(\sigma^{2}+\bar{\sigma}^{2}\right)$. Moreover, $\mathfrak{g} \cong \mathfrak{h}_{7}=(0,0,0,12,13,23)$.

(e) If $n=(4,5)$, there exists $\sigma$ in $P(\mathfrak{g}, J)$ such that $d \sigma^{3}=\sigma^{1} \bar{\sigma}^{2}+\sigma^{2} \bar{\sigma}^{1}$. The structure equations for $e(\sigma)$ are $(0,0,0,12,0,14-23)$ and so $\mathfrak{g} \cong \mathfrak{h}_{9}=(0,0,0,0,12,14+25)$.

(f) If $n=(3,5)$, then there exists $\sigma$ in $P(\mathfrak{g}, J)$ such that $d \sigma^{3}=(B-\bar{C}) \sigma^{1} \sigma^{2}+$ $B \sigma^{1} \bar{\sigma}^{2}+C \sigma^{2} \bar{\sigma}^{1}$. For all such $\sigma,(B-\bar{C})$ is non-zero. Moreover,

$$
\mathfrak{g} \cong\left\{\begin{array}{l}
\mathfrak{h}_{10}=(0,0,0,12,13,14), \\
\mathfrak{h}_{12}=(0,0,0,12,13,24), \\
\mathfrak{h}_{11}=(0,0,0,12,13,14+23) .
\end{array}\right.
$$

(g) If $n=(3,4)$, then

$$
\mathfrak{g} \cong\left\{\begin{array}{l}
\mathfrak{h}_{16}=(0,0,0,12,14,24) \\
\mathfrak{h}_{13}=(0,0,0,12,13+14,24) \\
\mathfrak{h}_{14}=(0,0,0,12,14,13+42) \\
\mathfrak{h}_{15}=(0,0,0,12,13+42,14+23)
\end{array}\right.
$$

Proof. The statements for $n=(6,6)$ and $(5,6)$ are elementary.

When $n=(4,6)$ the cases listed in (c) are the only possibilities given by the classification of [17].

When $n=(3,6)$, then $V_{1}=\left\langle\omega^{1}, \bar{\omega}^{1}, \omega^{2}+\bar{\omega}^{2}\right\rangle$ and $d \omega^{3} \in \Lambda^{2} V_{1}$. It follows that $d \omega^{3}=\rho \omega^{1}\left(\omega^{2}+\bar{\omega}^{2}\right)$ and so we have $(\mathrm{d})$.

If $n_{2}=5$, then $\epsilon=1$, and we may take $A=D=0$ as noted in the previous lemma. By (26), a complex number $u \neq 0$ exists so that $u d \omega^{3}+\bar{u} d \bar{\omega}^{3} \in \Lambda^{2} V_{1}$.

If in addition $n_{1}=3$, then $V_{1}=\left\langle\omega^{1}, \bar{\omega}^{1}, \omega^{2}+\bar{\omega}^{2}\right\rangle$. Taking $d \omega^{3}=\rho \omega^{1} \omega^{2}+B \omega^{1} \bar{\omega}^{2}+$ $C \omega^{2} \bar{\omega}^{1}$ gives $u \rho=u B-\bar{u} \bar{C}$. Now setting $\sigma^{1}=\omega^{1}, \sigma^{2}=\omega^{2}$ and $\sigma^{3}=u \omega^{3}$ puts the structure equations in the form (f). Note that if $B=\bar{C}$ in (f) then $d \sigma^{3}$ and $d \bar{\sigma}^{3}$ are linearly dependent and so $n_{1}=4$.

If, on the other hand, $n_{1}=4$ then $V_{1}=\left\langle\omega^{1}, \bar{\omega}^{1}, \omega^{2}+\bar{\omega}^{2}, \omega^{3}+\lambda \bar{\omega}^{3}\right\rangle$ for some $\lambda$. Then $\rho=0, B=\lambda \bar{C}$ and $C=\lambda \bar{B}$, since $d \omega^{3}+\lambda d \bar{\omega}^{3}=0$. In particular, $d \omega^{3}=B \omega^{1} \bar{\omega}^{2}+\lambda \bar{B} \omega^{2} \bar{\omega}^{1}$. This yields case (e).

The remaining case is $n=(3,4)$. Since this is the minimum possible combination for the invariant $n$, by exclusion all remaining nilpotent complex structures found in [16] and [17] are covered in this case. 
Corollary 14. Let $J$ be a nilpotent complex structure on a nilpotent Lie algebra $\mathfrak{g}$. Then complex structure is abelian if $n=(6,6),(5,6),(4,5)$. It is not abelian if $n_{1}=3$ and $n_{2}>4$.

3.4. More invariants of nilpotent complex structures. Given $\omega$ in $P(\mathfrak{g}, J)$. Suppose that its structure equations are (24). Let $\sigma$ be another element in $P(\mathfrak{g}, J)$. Viewing $\sigma$ and $\omega$ as row vectors, then $\sigma=\left(\sigma^{1}, \sigma^{2}, \sigma^{3}\right)$ and $\omega=\left(\omega^{1}, \omega^{2}, \omega^{3}\right)$ are related by a matrix: $\sigma^{j}=\sigma_{k}^{j} \omega^{k}$. This must be of the form

$$
\sigma(\omega):=\left(\begin{array}{ccc}
\sigma_{1}^{1} & \sigma_{1}^{2} & \sigma_{1}^{3} \\
\sigma_{2}^{1} & \sigma_{2}^{2} & \sigma_{2}^{3} \\
0 & 0 & \sigma_{3}^{3}
\end{array}\right),
$$

with $\epsilon \sigma_{2}^{1}=0$. So when $\epsilon \neq 0$ the matrix $\sigma(\omega)$ is upper triangular. Write $\Delta(\sigma, \omega)$ for the determinant of the transformation $\sigma(\omega)$, so that $\sigma^{1} \sigma^{2} \sigma^{3}=\Delta(\sigma, \omega) \omega^{1} \omega^{2} \omega^{3}$, and $\Delta(\sigma, \omega)^{-1}=\Delta(\omega, \sigma)$. Define $\Delta^{\prime}(\sigma, \omega)$ by $\sigma^{1} \sigma^{2}=\Delta^{\prime}(\sigma, \omega) \omega^{1} \omega^{2}$ so that $\Delta(\sigma, \omega)=$ $\sigma_{3}^{3} \Delta^{\prime}(\sigma, \omega)$. The space $\mathcal{A}$ of matrices as in (29) may be considered the automorphism group of the nilpotent complex structure, and $P(\mathfrak{g}, J)$ is the orbit of $\omega$ under the multiplication of elements in $\mathcal{A}$.

Consider the two functions $\Delta_{1}: P(\mathfrak{g}, J) \rightarrow \mathbb{C}, \Delta_{2}: P(\mathfrak{g}, J) \rightarrow \mathbb{R}$ defined respectively by

$$
\begin{aligned}
& d \sigma^{3} \wedge d \sigma^{3}=2 \Delta_{1}(\sigma) \sigma^{1} \bar{\sigma}^{1} \sigma^{2} \bar{\sigma}^{2}, \\
& d \sigma^{3} \wedge d \bar{\sigma}^{3}=2 \Delta_{2}(\sigma) \sigma^{1} \bar{\sigma}^{1} \sigma^{2} \bar{\sigma}^{2} .
\end{aligned}
$$

In terms of the structure constants for $\omega$,

$$
\begin{aligned}
& \Delta_{1}(\omega)=A D-B C \\
& \Delta_{2}(\omega)=\frac{1}{2}\left[|B|^{2}+|C|^{2}-A \bar{D}-\bar{A} D-|\rho|^{2}\right] .
\end{aligned}
$$

If $\sigma=\sigma(\omega)$ then

$$
\begin{aligned}
d \sigma^{3} \wedge d \sigma^{3} & =\left(\sigma_{3}^{3}\right) d \omega^{3} \wedge d \omega^{3}=\left(\sigma_{3}^{3}\right) \Delta_{1}(\omega) \omega^{1} \bar{\omega}^{1} \omega^{2} \bar{\omega}^{2} \\
& =\left(\sigma_{3}^{3}\right)^{2} \Delta_{1}(\omega)\left|\Delta^{\prime}(\omega, \sigma)\right|^{2} \sigma^{1} \bar{\sigma}^{1} \sigma^{2} \bar{\sigma}^{2}
\end{aligned}
$$

Therefore

$$
\Delta_{1}(\sigma)=\Delta_{1}(\omega)\left|\Delta^{\prime}(\omega, \sigma)\right|^{2}\left(\sigma_{3}^{3}\right)^{2}
$$

and similarly

$$
\Delta_{2}(\sigma)=\Delta_{2}(\omega)\left|\Delta^{\prime}(\omega, \sigma)\right|^{2}\left|\sigma_{3}^{3}\right|^{2} .
$$

By choosing $\sigma$ appropriately we may assume that $\Delta_{1}$ is either 0 or 1 . We observe that if $\Delta_{1}$ is non-zero in some basis then it is non-zero in every basis. In this situation $\Delta_{2} /\left|\Delta_{1}\right|$ is invariant under transformations of the form (29). Note that $\Delta_{2}^{2}-\left|\Delta_{1}\right|^{2}$ is scaled by a positive constant by an automorphism, so the sign of $\Delta_{2}^{2}-\left|\Delta_{1}\right|^{2}$ is another invariant. The significance of this can be seen as follows. Pick $\omega \in P$ and 
let $e=e(\omega)$ be the corresponding real basis. Then $d \omega^{3} \wedge d \omega^{3}=-8 \Delta_{1} e^{1234}$ and $d \omega^{3} \wedge d \bar{\omega}^{3}=-8 \Delta_{2} e^{1234}$, whence

$$
\begin{gathered}
d e^{5} \wedge d e^{5}=-4\left(\Delta_{2}+\operatorname{Re}\left(\Delta_{1}\right)\right) e^{1234} \\
d e^{6} \wedge d e^{6}=-4\left(\Delta_{2}-\operatorname{Re}\left(\Delta_{1}\right)\right) e^{1234} \\
d e^{5} \wedge d e^{6}=-4 \operatorname{Im}\left(\Delta_{1}\right) e^{1234}
\end{gathered}
$$

The numbers $\Delta_{2} \pm \operatorname{Re}\left(\Delta_{1}\right)$ determine whether or not the two-form $d e^{5}$ and $d e^{6}$ are simple or not. A two-form $\alpha$ is simple if and only if $\alpha \wedge \alpha=0$. The equation

$$
\left(s d e^{5}-t d e^{6}\right) \wedge\left(s d e^{5}-t d e^{6}\right)=0
$$

is equivalent to the second order homogeneous equation

$$
\left(\Delta_{2}+\operatorname{Re}\left(\Delta_{1}\right)\right) s^{2}-2 \operatorname{Im}\left(\Delta_{1}\right) s t+\left(\Delta_{2}-\operatorname{Re}\left(\Delta_{1}\right)\right) t^{2}=0 .
$$

As the discriminant of this equation is $\left|\Delta_{1}\right|^{2}-\Delta_{2}^{2}$, it has non-trivial real solutions if and only if $\left|\Delta_{1}\right|^{2}-\Delta_{2}^{2} \geq 0$.

If $d \omega^{3}$ and $d \bar{\omega}^{3}$ are linearly independent, a solution $(s, t)$ to $(36)$ exists precisely when $s d e^{5}+t d e^{6}$ is simple. When $\left|\Delta_{1}\right|^{2}-\Delta_{2}^{2}=0$ there is precisely one such nontrivial solution, when $\left|\Delta_{1}\right|^{2}-\Delta_{2}^{2}>0$ there are two. When $d \omega^{3}$ and $d \bar{\omega}^{3}$ are linearly dependent it is easy to see from equations (28), (32) and (33) that $\left|\Delta_{1}\right|^{2}=\Delta_{2}^{2}$.

3.5. Identification of underlying real algebras. Given the invariants of the last section, we now have the means to filter isomorphism classes of $\mathfrak{g}$ for a given set of structure constants $\epsilon, \rho, A, B, C, D$ of a nilpotent complex structure. As we determine the underlying real algebras, we also identify all the invariants in the complex structure equations in the next few paragraphs.

LEMMA 15. The following statements are equivalent.

(1) For every nilpotent complex structure $J$ on $\mathfrak{g}$ and every $\omega$ in $P(\mathfrak{g}, J)$, the condition $\Delta_{2}(\omega)=0=\Delta_{1}(\omega)$ holds.

(2) There exists a nilpotent $J$ on $\mathfrak{g}$ and some $\omega \in P(\mathfrak{g}, J)$ such that $\Delta_{2}(\omega)=0=$ $\Delta_{1}(\omega)$.

(3) The Lie algebra $\mathfrak{g}$ is isomorphic to one of the following

$$
\begin{aligned}
\mathfrak{h}_{1}=(0,0,0,0,0,0), \quad \mathfrak{h}_{8} & =(0,0,0,0,0,12), \quad \mathfrak{h}_{6}=(0,0,0,0,12,13), \\
\mathfrak{h}_{7}=(0,0,0,12,13,23), \quad \mathfrak{h}_{10} & =(0,0,0,12,13,14), \quad \mathfrak{h}_{16}=(0,0,0,12,14,24) .
\end{aligned}
$$

Proof. It is clear that (1) implies (2). Now suppose (2) holds: pick $J$ and $\omega$ so that $\Delta_{2}(\omega)=0=\Delta_{1}(\omega)$. Since $d \omega^{2}, d \bar{\omega}^{2}, d \omega^{3}, d \bar{\omega}^{3}$ span $d \mathfrak{g}_{\mathbb{C}}^{*}$ and $d \omega^{2} \wedge d \omega^{3}=0=d \omega^{2} \wedge d \bar{\omega}^{3}$ by the nilpotency of $J$, any two elements $\alpha_{1}, \alpha_{2}$ in $d \mathfrak{g}_{\mathbb{C}}^{*}$ satisfy $\alpha_{1} \wedge \alpha_{2}=0$. Since this is in particular also true for the real elements, a basis of simple two-forms for $d \mathfrak{g}^{*}$ exist so that any two basis elements satisfy $\alpha_{1} \wedge \alpha_{2}=0$. Now consult the classification of six dimensional nilpotent Lie algebras with complex structures [17, Theorem 2.9]. This gives (3). If (3) holds then any $\omega$ in $P(\mathfrak{g}, J)$ for any complex structure $J$ on $\mathfrak{g}$ has $d \omega^{i} \wedge d \omega^{j}=0=d \omega^{i} \wedge d \bar{\omega}^{j}$ for all $i, j$. This completes the proof.

Corollary 16. Suppose $\mathfrak{g}$ is not one of the Lie algebras listed in Lemma 15. For any integrable nilpotent $J$ and any $\omega$ in $P(\mathfrak{g}, J)$, one has $\Delta_{2}(\omega)^{2}+\left|\Delta_{1}(\omega)\right|^{2}>0$.

LEMMA 17. The following statements are equivalent. 
(1) For every nilpotent complex structure $J$ on $\mathfrak{g}$ and every $\omega$ in $P(\mathfrak{g}, J)$, the condition $\Delta_{2}(\omega)^{2}<\left|\Delta_{1}(\omega)\right|^{2}$ holds.

(2) There exists a nilpotent $J$ on $\mathfrak{g}$ and some $\omega$ in $P(\mathfrak{g}, J)$ such that the inequality $\Delta_{2}(\omega)^{2}<\left|\Delta_{1}(\omega)\right|^{2}$ is satisfied.

(3) The Lie algebra $\mathfrak{g}$ is isomorphic to one of the following

$\mathfrak{h}_{2}=(0,0,0,0,12,34), \quad \mathfrak{h}_{12}=(0,0,0,12,13,24), \quad \mathfrak{h}_{13}=(0,0,0,12,13+14,24)$.

Proof. The implication $(1) \Rightarrow(2)$ is trivial. Suppose that $J$ and $\omega$ are given as in (2). Solving (36), we get two real, simple two-forms in the span of $d \omega^{3}, d \bar{\omega}^{3}$. It follows that $d \mathfrak{g}^{*}$ has a basis consisting only of simple two-forms. The classification [17, Theorem 2.9], Lemma 15 and Corollary 16 give (3).

Now suppose that (3) holds and let $J$ be a nilpotent complex structure on $\mathfrak{g}$. Pick any $\omega$ in $P(\mathfrak{g}, J)$. Represent $\mathfrak{h}_{2}$ as $(0,0,0,0,13,24)$. For any of the three algebras listed, any nilpotent complex structure and any $\omega$ in $P(\mathfrak{g}, J)$, there are constants $a, b, c, r$ such that $d \omega^{3}=a e^{12}+b\left(e^{13}+r e^{14}\right)+c e^{24}$ where $r=0$ or 1 . So $d \omega^{3} \wedge d \omega^{3}=$ $-2 b c e^{1234}$ and $d \omega^{3} \wedge d \bar{\omega}^{3}=-(b \bar{c}+\bar{b} c) e^{1234}$. By Corollary $16, b c \neq 0$ so (after re-scaling of $\omega^{1}$ and $\omega^{2}$ ) we have $\left|\Delta_{1}\right|^{2}=|b \bar{c}|^{2} \geq \operatorname{Re}(b \bar{c})^{2}=\frac{1}{4}|b \bar{c}+\bar{b} c|^{2}=\Delta_{2}^{2}$. Equality occurs precisely if $b \bar{c}$ is real.

To see that this does not occur, note that by nilpotency of $J, d \omega^{2} \wedge d \omega^{2}=0=$ $d \omega^{2} \wedge d \omega^{3}$, whence $d \omega^{2}=u e^{12}$ for some complex number $u$. If $u=0$ then $\mathfrak{g}=\mathfrak{h}_{2}$ and $a=r=0$. Otherwise, take $u=1$ and $\omega^{3}-\omega^{2}$ as a 'new' $\omega^{3}$. This has $a=0$. So for all three algebras and all $J$, we can take an $\omega$ in $P(\mathfrak{g}, J)$ with $a=0$. Then $d \omega^{3}$ and $d \bar{\omega}^{3}$ are linearly dependent precisely when $b \bar{c}$ is real. In this case $n_{1}$ is 4 if $\epsilon \neq 0$, and 5 otherwise. The latter value is not realized for the given algebras. Only $(0,0,0,0,13,24)$ has $n_{1}=4$ but clearly $d \omega^{2} \wedge d \omega^{2}=0=d \omega^{2} \wedge d \omega^{3}$ shows that for this algebra $\epsilon=0$ for all $J$. Therefore $b \bar{c}$ is never real and so $\left|\Delta_{1}\right|^{2}>\Delta_{2}^{2} \square$

LEMMA 18. The following statements are equivalent.

(1) For every nilpotent complex structure $J$ on $\mathfrak{g}$ and every $\omega \in P(\mathfrak{g}, J)$, the condition $\Delta_{2}(\omega)^{2}>\left|\Delta_{1}(\omega)\right|^{2}$ holds.

(2) There exists a nilpotent $J$ on $\mathfrak{g}$ and some $\omega \in P(\mathfrak{g}, J)$ such that the inequality $\Delta_{2}(\omega)^{2}>\left|\Delta_{1}(\omega)\right|^{2}$ is satisfied.

(3) The Lie algebra $\mathfrak{g}$ is isomorphic to one of the following

$$
\mathfrak{h}_{5}=(0,0,0,0,13+42,14+23), \quad \mathfrak{h}_{15}=(0,0,0,12,13+42,14+23) .
$$

Proof. The idea is as for the preceding Lemmas. Suppose (2). There are then no simple elements in the real span of $d \omega^{3}+d \bar{\omega}^{3}, i\left(d \omega^{3}-d \bar{\omega}^{3}\right)$ as equation (36) has no real solutions. This of course means that for the real basis $e(\omega)$ all linear combinations of $d e^{5}$ and $d e^{6}$ are non-simple. In [17, Theorem 2.9] only two algebras have the property that all elements in the span of $\left\{d e^{i}\right\}$ are non-simple. These are listed in (3).

Building a nilpotent $J$ from $\mathfrak{h}_{5}$ or $\mathfrak{h}_{15}$ gives $d \omega^{3}=a e^{12}+b\left(e^{13}+e^{42}\right)+c\left(e^{14}+e^{23}\right)$. Then $\Delta_{1}=b^{2}+c^{2}$ and $\Delta_{2}^{2}=|b|^{2}+|c|^{2}$, so $\Delta_{2}^{2}-\left|\Delta_{1}\right|^{2}=2\left(|b \bar{c}|^{2}-\operatorname{Re}(b \bar{c})^{2}\right)=2 \operatorname{Im}(b \bar{c}) \geq$ 0 , with equality if and only if $b \bar{c}$ is real. Arguing as in the proof of Lemma 17 one shows that $b \bar{c}$ cannot be real. It proves the implications (2) to (3). The implication (1) to (2) is obvious. 
Now one case is left, namely $\left|\Delta_{1}\right|^{2}=\Delta_{2}^{2}>0$. By Lemmas 15,17 and 18 this condition must characterize the remaining algebras in the classification of Lemma 13. In one special case we may be more explicit.

Lemma 19. Suppose $\omega$ in $P(\mathfrak{g}, J)$ is such that $\left|\Delta_{1}\right|^{2}=\Delta_{2}^{2}>0$ and $d=1$. Then there exists $\sigma$ in $P(\mathfrak{g}, J)$ such that the real basis $e(\sigma)$ has the following structure equations

- $\mathfrak{h}_{3}=\left(0,0,0,0,0,12-\operatorname{Sign}\left(\Delta_{2}\right) 34\right)$ if $\epsilon=0,\left|\Delta_{1}\right|^{2}=\Delta_{2}^{2}>0$,

- $\mathfrak{h}_{9}=(0,0,0,12,0,14-23)$ if $\epsilon=1$ and $\left|\Delta_{1}\right|^{2}=\Delta_{2}^{2}>0$.

Proof. Suppose that $\epsilon=0$ and $\left|\Delta_{1}\right|^{2}=\Delta_{2}^{2}>0$. Note that $\Delta_{2}=|C|^{2}-\bar{A} D$ by (28). Define $\lambda>0$ by $\Delta_{2}=\operatorname{Sign}\left(\Delta_{2}\right) \lambda^{2}$. Then

$$
\bar{A} d \omega^{3}=\left(A \omega^{1}+C \omega^{2}\right)\left(\bar{A} \bar{\omega}^{1}+\bar{C} \bar{\omega}^{2}\right)-\operatorname{Sign}\left(\Delta_{2}\right) \lambda^{2} \omega^{2} \bar{\omega}^{2},
$$

which gives that second part if $A \neq 0$. If $D \neq 0$ we rewrite similarly.

If $A=0=D, \Delta_{2}=|B|^{2}=|C|^{2}>0$. We pick square roots of $B$ and $C$, and set

$$
\sigma^{1}=\frac{1}{\sqrt{2}}\left(\sqrt{\frac{B}{C}} \omega^{1}+\omega^{2}\right), \quad \sigma^{2}=\frac{1}{\sqrt{2}}\left(-\omega^{1}+\sqrt{\frac{C}{B}} \omega^{2}\right), \quad \sigma^{3}=-\frac{1}{2 \sqrt{B C}} \omega^{3} .
$$

Then $d \sigma^{3}=-(1 / 2)\left(\sigma^{1} \bar{\sigma}^{1}-\sigma^{2} \bar{\sigma}^{2}\right)$, whence $d e^{6}=e^{12}-e^{34}$.

If $\epsilon=1$ then $A=D=0$. If $\left|\Delta_{1}\right|^{2}=\Delta_{2}^{2}>0$, we take

$$
\sigma^{1}=2 \sqrt{\frac{B}{C}} \omega^{1}, \quad \sigma^{2}=-2 \omega^{2}, \quad \sigma^{3}=\frac{2}{\sqrt{B C}} \omega^{3}
$$

to get $d \sigma^{1}=0, d \sigma^{2}=-(1 / 2) \sigma^{2}, d \sigma^{3}=-(1 / 2)\left(\sigma^{1} \bar{\sigma}^{2}+\sigma^{2} \bar{\sigma}^{1}\right)$.

Lemma 20. Suppose $\omega$ in $P(\mathfrak{g}, J)$ is such that $\left|\Delta_{1}\right|^{2}=\Delta_{2}^{2}>0, d \omega^{3}$ and $d \bar{\omega}^{3}$ are linearly independent. Then $\mathfrak{g}$ is one of $\mathfrak{h}_{4}, \mathfrak{h}_{11}, \mathfrak{h}_{14}$, with $n_{2}$ being an invariant to distinguish the different spaces.

Proof. The proof is similar to the one of the last lemma. As this is the last remaining case in the classification of all nilpotent complex structures, one may also identify the concerned algebras using [16] or [17]. $\square$

Next, we tabulate the invariants for all nilpotent complex structures according to their underlying nilpotent algebras.

THEOREM 21. A nilpotent complex structure on a six-dimensional nilpotent Lie algebra $\mathfrak{g}$ is determined by the data of its nilpotent complex structures, and vice-versa, as indicated in Table 3.1 below.

REMARK 1. It is known that each of the four algebras with a ' $*$ ' in the $|\rho|$ column admits both abelian and non-abelian complex structures [17]. For $\mathfrak{h}_{5}$ and $\mathfrak{h}_{15}$ this is particularly easy to see as both may be represented with either $d \omega^{3}=\omega^{1} \omega^{2}$ or $d \omega^{3}=\omega^{1} \bar{\omega}^{2}$. An abelian complex structures on $\mathfrak{h}_{2}$ is given by $d \omega^{3}=i \omega^{1} \bar{\omega}^{1}+\omega^{2} \bar{\omega}^{2}$ and on $\mathfrak{h}_{4}$ by $d \omega^{3}=i \omega^{1} \omega^{1}+\omega^{1} \bar{\omega}^{2}+\omega^{2} \bar{\omega}^{2}$. A non-abelian nilpotent complex structure on $\mathfrak{h}_{2}$ and $\mathfrak{h}_{4}$ may be obtained for instance by setting $d \omega^{1}=0=d \omega^{2}$ and $d \omega^{3}=$ $\rho \omega^{1} \omega^{2}+B \omega^{1} \bar{\omega}^{2}+B^{-1} \omega^{2} \bar{\omega}^{1}$ for some $B$ such that $|B| \neq 1$ with $|\rho|^{2}=\left(|B| \pm\left|B^{-1}\right|\right)^{2}$ for $\mathfrak{h}_{4}$ and $\left(|B|-\left|B^{-1}\right|\right)^{2}<|\rho|^{2}<\left(|B|+\left|B^{-1}\right|\right)^{2}$ for $\mathfrak{h}_{2}$. We note that any other choice of $\rho$ gives a non-abelian complex structure on $\mathfrak{h}_{5}$ and one on $\mathfrak{h}_{15}$ if we take $d \omega^{2}=\omega^{1} \bar{\omega}^{1}$ instead. 


\begin{tabular}{|l|l|c|c|c|c|c|c|}
\hline$n$ & $\mathfrak{g}$ & $\left|\Delta_{1}\right|^{2}-\Delta_{2}^{2}$ & $\left|\Delta_{1}\right|$ & $\left|\Delta_{2}\right|$ & $\epsilon$ & $|\rho|$ & $d$ \\
\hline$(6,6)$ & $\mathfrak{h}_{1}=(0,0,0,0,0,0)$ & 0 & 0 & 0 & 0 & 0 & 0 \\
$(5,6)$ & $\mathfrak{h}_{8}=(0,0,0,0,0,12)$ & 0 & 0 & 0 & 0 & 0 & 1 \\
$(5,6)$ & $\mathfrak{h}_{3}=(0,0,0,0,0,12+34)$ & 0 & + & + & 0 & 0 & 1 \\
$(4,6)$ & $\mathfrak{h}_{6}=(0,0,0,0,12,13)$ & 0 & 0 & 0 & 0 & + & 2 \\
$(4,6)$ & $\mathfrak{h}_{4}=(0,0,0,0,12,14+23)$ & + & + & + & 0 & $*$ & 2 \\
$(4,6)$ & $\mathfrak{h}_{2}=(0,0,0,0,12,34)$ & - & $*$ & + & 0 & $*$ & 2 \\
$(4,6)$ & $\mathfrak{h}_{5}=(0,0,0,0,13+42,14+23)$ & 0 & + & + & 1 & 0 & 1 \\
\hline$(4,5)$ & $\mathfrak{h}_{9}=(0,0,0,0,12,14+25)$ & 0 & 0 & 0 & 1 & + & 2 \\
$(3,6)$ & $\mathfrak{h}_{7}=(0,0,0,12,13,23)$ & 0 & 0 & 0 & 1 & + & 2 \\
$(3,5)$ & $\mathfrak{h}_{10}=(0,0,0,12,13,14)$ & 0 & + & + & 1 & + & 2 \\
$(3,5)$ & $\mathfrak{h}_{11}=(0,0,0,12,13,14+23)$ & 0 & + & $*$ & 1 & + & 2 \\
$(3,5)$ & $\mathfrak{h}_{12}=(0,0,0,12,13,24)$ & + & + & $*$ & 1 & + & 2 \\
$(3,4)$ & $\mathfrak{h}_{16}=(0,0,0,12,14,24)$ & 0 & + & + & 1 & + & 2 \\
$(3,4)$ & $\mathfrak{h}_{13}=(0,0,0,12,13+14,24)$ & - & $*$ & + & 1 & $*$ & 2 \\
$(3,4)$ & $\mathfrak{h}_{14}=(0,0,0,12,14,13+24)$ & + & + \\
$(3,4)$ & $\mathfrak{h}_{15}=(0,0,0,12,13+24,14+23)$ & - & & + & \\
\hline
\end{tabular}

TABLE 3.1

$\mathfrak{g}$ and parameters in the complex structure equations.

In the table, '0', '+' and '-' indicates that the value of the corresponding number is zero, positive or negative, while '*' means that the value is constrained only by the data to its left in the table. The number $d$ of the right-most column is the dimension of the linear span of $d \omega^{3}$ and $d \bar{\omega}^{3}$.

For the algebras with *'s in the other columns, i.e. those with different values of $\left|\Delta_{1}\right|^{2}, \Delta_{2}^{2}$, it is also always possible to find a complex structure such that the smaller of the two is zero.

Lemma 22. If $d=2, \Delta_{1}(\omega)=0=\Delta_{2}(\omega)$, then the complex structure is nonabelian.

Furthermore, $\epsilon=0$ if and only if there exists $\sigma$ in $P(\mathfrak{g}, J)$ such that $e(\sigma)$ has structure equations $\mathfrak{h}_{6}=(0,0,0,0,13,14)$. When $\epsilon=1$, one of the following three cases occurs.

- If there exists an $\omega$ in $P(\mathfrak{g}, J)$ such that $B=0$, there exists a $\sigma$ such that the equation for appropriate $e(\sigma)$ is $\mathfrak{h}_{10}=(0,0,0,12,13,14)$.

- If there exists an $\omega$ in $P(\mathfrak{g}, J)$ such that $B / \rho>0$, a $\sigma$ may be chosen such that the equation for appropriate $e(\sigma)$ is $\mathfrak{h}_{7}=(0,0,0,12,13,23)$.

- Otherwise $\sigma$ may be chosen such that the equation for appropriate $e(\sigma)$ the structure equations are $\mathfrak{h}_{16} \cong(0,0,-t(12), s(12), 13,23)$ and $(s+i t)^{2}=B / \rho$.

Proof. A simple exercise in algebra using the expressions (32) and (33), Lemma 13 and Lemma 12(f) shows that if $\Delta_{1}(\omega)=0=\Delta_{2}(\omega)$ and $\rho=0$ then $d \omega^{3}$ and $d \bar{\omega}^{3}$ are linearly dependent. This gives the first statement.

Suppose that $\epsilon=0$. If in addition $A=0$, then $\Delta_{1}=-B C=0$. When $B=0$, $\triangle_{2}=0$ gives $|C|^{2}=|\rho|^{2}$. Then we may rearrange to get

$$
d \omega^{3}=\left(\rho\left(\omega^{1}+(\bar{D} / \bar{C}) \omega^{2}\right)-C\left(\bar{\omega}^{1}+(D / C) \bar{\omega}^{2}\right)\right) \omega^{2} .
$$

So choose $r, c$ such that $r^{2}=\rho$ and $c^{2}=-C$ and set $\sigma^{1}=(r / c)\left(\omega^{1}+(\bar{D} / \bar{C}) \omega^{2}\right)$, 
$\sigma^{2}=2 \omega^{2}$ and $\sigma^{3}=\omega^{3} /(c r)$ to get $d \sigma^{3}=\frac{1}{2}\left(\sigma^{1}+\bar{\sigma}^{1}\right) \sigma^{2}$. If $C=0$, we note that

$$
d \omega^{3}=\left(\omega^{1}+(D / B) \omega^{2}\right)\left(\rho \omega^{2}+B \bar{\omega}^{2}\right) .
$$

Take $r, b$ such that $r^{2}=\rho$ and $b^{2}=B$ and set $\sigma^{1}=-(r / c) \omega^{2}, \sigma^{2}=2\left(\omega^{1}+\right.$ $\left.(D / B) \omega^{2}\right), \sigma^{3}=\omega^{3} /(b c)$. Then $d \sigma^{3}=\frac{1}{2}\left(\sigma^{1}+\bar{\sigma}^{1}\right) \sigma^{2}$, again. If $D=0$ instead of $A=0$, we interchange $\omega^{1}$ and $\omega^{2}$ and proceed with an argument as above.

Finally, if $A D=B C \neq 0$, we may write

$d \omega^{3}=\left(\rho|C|^{2}\left(|C|^{2}-\bar{A} D\right)^{-1}\left(\omega^{1}+(\bar{D} / \bar{C}) \omega^{2}\right)-C\left(\bar{\omega}^{1}+(D / C) \bar{\omega}^{2}\right)\right)\left((A / C) \omega^{1}+\omega^{2}\right)$.

Since $0<|\rho|^{2}=\left.|| C\right|^{2}-\left.\bar{A} D\right|^{2} /|C|^{2}$ this is equivalent to $d \sigma^{3}=\frac{1}{2}\left(\sigma^{1}+\bar{\sigma}^{1}\right) \sigma^{2}$.

When $\epsilon=1$, then $A=0=D$. As $\Delta_{1}=0$, by definition (32) $B C=0$. If $B=0$, then $d \omega^{3}=\left(\rho \omega^{1}-C \bar{\omega}^{1}\right) \omega^{2}$, which we may treat precisely as above to get $d \sigma^{1}=0, d \sigma^{2}=-\frac{1}{2} \sigma^{1} \bar{\sigma}^{1}, d \sigma^{3}=\frac{1}{2}\left(\sigma^{1}+\bar{\sigma}^{1}\right) \sigma^{2}$. If $C=0$, pick square roots: $r^{2}=$ $\rho, b^{2}=B$ and set $\sigma^{1}=\omega^{1}, \sigma^{2}=-\frac{1}{2}(r / b) \omega^{2}, \sigma^{3}=\frac{1}{2} \omega^{3}$. Then $d \sigma^{3}=\sigma^{1}\left(\sigma^{2}+\bar{\sigma}^{2}\right)$ but $d \sigma^{2}=(r / b) \sigma^{1} \bar{\sigma}^{1}$. Writing $r / b=s+i t$ we get

$$
d e^{1}=0, \quad d e^{2}=0, \quad d e^{3}=-t e^{12}, \quad d e^{4}=s e^{12}, \quad d e^{5}=e^{13}, \quad d e^{6}=e^{23} .
$$

When $r / b$ is real (which happens if and only if $\rho / B>0$ ) this is precisely $(0,0,0,12,13,23)$. When $r / b$ is purely imaginary, we get $(0,0,12,0,13,23) \cong$ $\mathfrak{h}_{16}$. Otherwise, replace $e^{4}$ by $s e^{3}+t e^{4}$ and divide $e^{3}, e^{5}$ and $e^{6}$ with $-t$ to get $(0,0,12,0,13,23)$ again.

COROllary 23. There are no abelian complex structures on $\mathfrak{h}_{p}$ for $p=$ $6,7,10,11,12,13,14,16$. Moreover, suppose that $\omega$ in $P(\mathfrak{g}, J)$ has structure constants $\epsilon, \rho, A, B, C, D$. If $\epsilon=0=\rho$ and $\Delta_{1}=0$, then $\Delta_{2} \geq 0$ with $\Delta_{2}=0$ if and only if d $\omega^{3}$ and $d \bar{\omega}^{3}$ are linearly dependent. If $\epsilon=1$ and $\rho=0$ then $\Delta_{2}^{2}-\left|\Delta_{1}\right|^{2} \geq 0$ with equality if and only if $d \omega^{3}$ and $d \bar{\omega}^{3}$ are linearly dependent.

Proof. For $p=7,10,11$ and 12 this was established by Lemma 13. For $p=6$ and 16 , any complex structure on $\mathfrak{h}_{p}$ has $\Delta_{1}=0=\Delta_{2}$ by Lemma 15 . However, $\Delta_{1}=0$ with $\rho=0=\epsilon$ implies $\Delta_{2} \geq 0$ with equality if and only if $d \omega^{3}$ and $d \bar{\omega}^{3}$ are linearly dependent. For $p=13$ and $14, \epsilon=1$ and $\left|\Delta_{1}\right|^{2} \geq \Delta_{2}^{2}$. The first statement may then be seen to follow from the second and third.

If $\epsilon=0=\rho=\Delta_{1}=0$ then clearly

$$
2 \Delta_{2}= \begin{cases}|B|^{2}+|C|^{2}, & \text { if } A=0 \\ |\bar{A} B-A \bar{C}|^{2} /|A|^{2}, & \text { if } A \neq 0\end{cases}
$$

In either case $\Delta_{2} \geq 0$. If $\Delta_{2}=0, d \omega^{3}=D \omega^{2} \bar{\omega}^{2}$ in the first case, and $\bar{A} B=A \bar{C}$ in the second. It is now easy to see that the equations of Lemma 12(f) are satisfied in either case.

If $\epsilon=1$ and $\rho=0$

$$
\Delta_{2}^{2}-\left|\Delta_{1}\right|^{2}=\left(|B|^{2}-|C|^{2}\right) \geq 0
$$

so equality implies $|B|=|C|$. Since we may assume that $A=0$ when $\epsilon=1$ this shows that $d \omega^{3}$ and $d \bar{\omega}^{3}$ are linearly dependent via Lemma $12(\mathrm{f})$. $\square$ 
4. Classification of $\operatorname{DGA}(\mathfrak{g}, J)$. In this section we calculate the isomorphism class of the six-dimensional complex Lie algebras $\mathfrak{f}^{1}=\mathfrak{f}^{1}(\mathfrak{g}, J)$ obtained from a nilpotent algebra $\mathfrak{g}$ equipped with a complex structure $J$. Our aim is to identify the complex Lie algebra structure of $\mathfrak{f}^{1}$ for a given $\mathfrak{g}$ and $J$. The result will identify $\mathfrak{f}^{1}$ as the complexification of one of the real nilpotent algebras $\mathfrak{h}_{n}$.

When a complex structure $J$ is given, recall that the Lie algebra structure on $\bar{f}^{1}$ is defined by $\bar{\partial}: \mathfrak{g}^{(1,0)} \oplus \mathfrak{g}^{*(0,1)} \rightarrow \Lambda^{2}\left(\mathfrak{g}^{(1,0)} \oplus \mathfrak{g}^{*(0,1)}\right)$. If $X \in \mathfrak{g}^{(1,0)}, \bar{Y} \in \mathfrak{g}^{(0,1)}, \omega \in$ $\mathfrak{g}^{*(0,1)}$, then $\bar{\partial} \omega$ is the $(2,0)$-component of $d \omega$ and $(\bar{\partial} X)(\bar{Y})$ is the $(1,0)$-part of the vector $-[X, \bar{Y}]^{1,0}$. Let $T_{1}, T_{2}, T_{3}$ be dual to $\omega^{1}, \omega^{2}, \omega^{3}$. Given the equations (24), the differential $\bar{\partial}$ is determined by the following structure equations.

$$
\left\{\begin{array}{l}
\bar{\partial} \bar{\omega}^{1}=0, \quad \bar{\partial} \bar{\omega}^{2}=0, \quad \bar{\partial} \bar{\omega}^{3}=\rho \bar{\omega}^{12}, \\
\bar{\partial} T_{1}=\epsilon \bar{\omega}^{1} T_{2}+\left(A \bar{\omega}^{1}+B \bar{\omega}^{2}\right) T_{3}, \quad \bar{\partial} T_{2}=\left(C \bar{\omega}^{1}+D \bar{\omega}^{2}\right) T_{3}, \quad \bar{\partial} T_{3}=0 .
\end{array}\right.
$$

The Schouten bracket is an extension of the following Lie bracket on $\mathfrak{f}^{1}$.

$$
\left\{\begin{array}{l}
{\left[T_{1} \bullet T_{2}\right]=-\rho T_{3},} \\
{\left[T_{1} \bullet \bar{\omega}^{2}\right]=-\epsilon \bar{\omega}^{1}, \quad\left[T_{1} \bullet \bar{\omega}^{3}\right]=-\bar{A} \bar{\omega}^{1}-\bar{C} \bar{\omega}^{2}, \quad\left[T_{2} \bullet \bar{\omega}^{3}\right]=-\bar{B} \bar{\omega}^{1}-\bar{D}^{2} \bar{\omega}^{2} .}
\end{array}\right.
$$

In this section, we ignore at first the Lie algebra structure on $\mathfrak{f}^{1}$ and focus on the differential structure $\bar{\partial}$ of $\mathfrak{f}^{1}$ seen as a differential graded algebra. Inspecting the differential algebra structure, we identify the Lie algebra structure of $\left(\mathfrak{f}^{1}\right)^{*} \cong \bar{f}^{1}$ as the complexification of $\mathfrak{h}_{n}$ for some $n$. Taking complex conjugation, we recover the Lie algebra structure on $\mathfrak{f}^{1}$ as a complexification of the same $\mathfrak{h}_{n}$. The results are presented in Table 4.1.

In the presentation below, the subscript $\mathbb{C}$ in the identification $\mathfrak{f}^{1} \cong\left(\mathfrak{h}_{n}\right)_{\mathbb{C}}$ is suppressed.

Change basis by setting

$$
\left(\eta^{1}, \eta^{2}, \eta^{3}, \eta^{4}, \eta^{5}, \eta^{6}\right):=\left(\bar{\omega}^{1}, T_{3}, \bar{\omega}^{2}, T_{2}, \bar{\omega}^{3}, T_{1}\right) .
$$

This gives the following structure equations

$$
\begin{aligned}
\bar{\partial} \eta_{1}=\bar{\partial} \eta_{2}=\bar{\partial} \eta_{3}=0, & \bar{\partial} \eta^{4}=C \eta^{12}+D \eta^{32} \\
\bar{\partial} \eta^{5}=\rho \eta^{13}, & \bar{\partial} \eta^{6}=\epsilon \eta^{14}+A \eta^{12}+B \eta^{32}
\end{aligned}
$$

which clearly define a complex 6-dimensional nilpotent Lie algebra.

When the invariants $\epsilon, \rho, \Delta_{1}$ and $\Delta_{2}$ are given, we shall use the complex structure equations (40) to identify the Lie algebra underlying $\bar{f}^{-1}$ and hence $\mathfrak{f}^{1}$. On the other hand, we use the invariants and the classification in Table 3.1 to identify the originating Lie algebra $\mathfrak{g}$. These are listed in the right most column of Table 4.1.

4.1. The cases when $\epsilon=0$. By Corollary $14, n_{2}=6$. Then the potentially non-zero structure equations are

$$
\bar{\partial} \eta^{4}=C \eta^{12}+D \eta^{32}, \quad \bar{\partial} \eta^{5}=\rho \eta^{13}, \quad \bar{\partial} \eta^{6}=A \eta^{12}+B \eta^{32} .
$$

There are six possibilities depending on the rank of $X:=\left(\begin{array}{ll}A & B \\ C & D\end{array}\right)$ and $\rho$. 


\subsubsection{When $\rho=0$.}

(1) If $\operatorname{rank} X=0$ then $\Delta_{1}=0$ and $\Delta_{2}=0$. It follows that $\mathfrak{f}_{1} \cong \mathfrak{h}_{1}$ and $\mathfrak{g}=\mathfrak{h}_{1}$.

(2) If the rank of $X$ is one then $\Delta_{1}=0, \Delta_{2} \geq 0$ and $\mathfrak{f}^{1} \cong \mathfrak{h}_{8}$. By Corollary $23, d \omega^{3}$ and $d \bar{\omega}^{3}$ are linearly dependent if and only if $\Delta_{2}=0$. Therefore, by Table 3.1 $\mathfrak{g}=\mathfrak{h}_{8}$ when $\Delta_{2}=0$, and $\mathfrak{g}=\mathfrak{h}_{5}$ when $\Delta_{2} \neq 0$.

(3) If rank $X=2$ and $\rho=0$ then $\Delta_{1} \neq 0, \Delta_{2}$ is unconstrained and $\mathfrak{f}^{1} \cong \mathfrak{h}_{6}$. By Table $3.1, \mathfrak{g}=\mathfrak{h}_{2}, \mathfrak{h}_{3}, \mathfrak{h}_{4}$ or $\mathfrak{h}_{5}$.

This case accounts for the first four items in Table 4.1.

\subsubsection{When $\rho \neq 0$.}

(1) If $\operatorname{rank} X=0$ then $\Delta_{1}=0$ and $\Delta_{2}>0$. It follows that $\mathfrak{f}_{1} \cong \mathfrak{h}_{8}$ and $\mathfrak{g}=\mathfrak{h}_{5}$.

(2) If $\operatorname{rank} X=1$ then $\Delta_{1}=0$ and $\Delta_{2}$ is unconstrained. Then $\mathfrak{f}^{1} \cong \mathfrak{h}_{6}$. However, when the value of $\Delta_{2}$ varies from zero to non-zero, the algebra $\mathfrak{g}$ changes from $\mathfrak{h}_{5}$ to $\mathfrak{h}_{6}$.

(3) If $\operatorname{rank} X=2$ then $\Delta_{1} \neq 0, \Delta_{2}$ is unconstrained and $\mathfrak{f}^{1} \cong \mathfrak{h}_{7}$. The invariants $\left|\Delta_{2}\right|$ and $\left|\Delta_{1}\right|^{2}-\Delta_{2}^{2}$ help to identify the three possibilities $\mathfrak{h}_{2}, \mathfrak{h}_{4}, \mathfrak{h}_{5}$ for the algebra $\mathfrak{g}$.

4.2. The cases when $\epsilon \neq 0$. We assume that $\epsilon=1, A=D=0$. Then the potentially non-zero structure equations are

$$
\bar{\partial} \eta^{4}=C \eta^{12}, \quad \bar{\partial} \eta^{5}=\rho \eta^{13}, \quad \bar{\partial} \eta^{6}=\eta^{14}+B \eta^{32} .
$$

4.2.1. When $\rho=0$. There are three cases (discarding $B=0=C$ ).

(1) If $C=0$ then $\Delta_{1}=0, \Delta_{2}>0$. It follows that $\mathfrak{f}^{1} \cong \mathfrak{h}_{3}$ and $\mathfrak{g}=\mathfrak{h}_{15}$.

(2) If $B=0$ then $\Delta_{1}=0$ and $\Delta_{2}>0$. Then $\mathfrak{f}^{1} \cong \mathfrak{h}_{17}=(0,0,0,0,12,15)$ and $\mathfrak{g}=\mathfrak{h}_{15}$.

(3) If $B C \neq 0$ then $\mathfrak{f}^{1} \cong \mathfrak{h}_{9}$. As $\Delta_{1} \neq 0$, by Corollary $23, \Delta_{2}^{2}-\left|\Delta_{1}\right|^{2} \geq 0$ with equality if and only if $d \omega^{3}$ and $d \bar{\omega}^{3}$ are linearly dependent. It yields two algebras for $\mathfrak{g}$, namely $\mathfrak{h}_{9}$ and $\mathfrak{h}_{15}$.

4.2.2. When $\rho \neq 0$. There are four cases for $\mathfrak{f}^{1}$ :

(1) If $B=0=C$ then $\mathfrak{f}^{1} \cong \mathfrak{h}_{6}$. As $\Delta_{1}=0, \Delta_{2}<0$, and $\mathfrak{g}=\mathfrak{h}_{15}$.

(2) If $C=0, B \neq 0$ then $\mathfrak{f}^{1} \cong \mathfrak{h}_{4}$. As $\Delta_{1}=0$ but $\Delta_{2}$ is unconstrained, by Table 3.1, $\mathfrak{g}$ could be one of $\mathfrak{h}_{7}, \mathfrak{h}_{16}$ or $\mathfrak{h}_{15}$.

(3) If $B=0, C \neq 0$, then $\mathfrak{f}^{1} \cong \mathfrak{h}_{10}$. As $\Delta_{1}=0, \Delta_{2}$ is unconstrained, we get $\mathfrak{g}=\mathfrak{h}_{10}$ if $\Delta_{2}=0$. Otherwise, we get $\mathfrak{g}=\mathfrak{h}_{15}$.

(4) If $B C \neq 0$ then $\mathfrak{f}^{1} \cong \mathfrak{h}_{11} . \Delta_{1} \neq 0, \Delta_{2}$ is unconstrained. An inspection of Table 3.1 yields the five different algebras $\mathfrak{h}_{11}, \mathfrak{h}_{12}, \mathfrak{h}_{13}, \mathfrak{h}_{14}$ and $\mathfrak{h}_{15}$.

To recap all the computations, we have used the invariants of the complex structural equations to identity both the underlying real Lie algebra and the structure of the Lie algebra $\mathfrak{f}^{1}$. At the cost of being repetitive, we recall in the following how the invariants are defined.

THEOREM 24. Suppose that $\mathfrak{g}$ is a real six-dimensional nilpotent algebra with a nilpotent complex structure $J$. Then there exists a basis $\omega^{1}, \omega^{2}, \omega^{3}$ for $\mathfrak{g}^{*(1,0)}$ such that

$$
\left\{\begin{array}{l}
d \omega^{1}=0, \quad d \omega^{2}=\epsilon \omega^{1} \bar{\omega}^{1}, \\
d \omega^{3}=\rho \omega^{1} \omega^{2}+A \omega^{1} \bar{\omega}^{1}+B \omega^{1} \bar{\omega}^{2}+C \omega^{2} \bar{\omega}^{1}+D \omega^{2} \bar{\omega}^{2},
\end{array}\right.
$$

where $\epsilon, \rho \in\{0,1\}$. Moreover, let

$$
\begin{aligned}
& \triangle_{1}=A D-B C ; \quad \triangle_{2}=\frac{1}{2}\left[|B|^{2}+|C|^{2}-A \bar{D}-\bar{A} D-|\rho|^{2}\right] ; \\
& d=\operatorname{dim}_{\mathbb{C}}\left\langle d \omega^{3}, d \bar{\omega}^{3}\right\rangle, \quad X=\left(\begin{array}{l}
A \\
C
\end{array}\right)
\end{aligned}
$$


be the invariants associated to the structure equations. Given a real algebra $\mathfrak{g}$ in the right-most column, Table 4.1 lists constraints on the values of the invariants that can be realized by a complex structure $J$ on $\mathfrak{g}$, as well as the relevant isomorphism class of the Lie algebra $\mathfrak{f}^{1}$ in the left-most column. A “*” indicates an un-constrained invariant.

\begin{tabular}{|l|c|c|c|c|c|c|c|c|c|c|}
\hline $\mathfrak{f}^{1}$ & $\epsilon$ & $|\rho|$ & $\operatorname{rank} X$ & $\left|\Delta_{1}\right|$ & $\left|\Delta_{2}\right|$ & $\left|\Delta_{1}\right|^{2}-\Delta_{2}^{2}$ & $d$ & $|B|$ & $|C|$ & $\mathfrak{g}$ \\
\hline $\mathfrak{h}_{1}$ & 0 & 0 & 0 & 0 & 0 & 0 & 0 & 0 & 0 & $\mathfrak{h}_{1}$ \\
$\mathfrak{h}_{8}$ & 0 & 0 & 1 & 0 & 0 & 0 & 1 & $*$ & $*$ & $\mathfrak{h}_{8}$ \\
$\mathfrak{h}_{8}$ & 0 & 0 & 1 & 0 & + & - & 2 & $*$ & $*$ & $\mathfrak{h}_{5}$ \\
$\mathfrak{h}_{6}$ & 0 & 0 & 2 & + & $*$ & - & 2 & $*$ & $*$ & $\mathfrak{h}_{2}, \mathfrak{h}_{3}, \mathfrak{h}_{4}, \mathfrak{h}_{5}$ \\
\hline $\mathfrak{h}_{8}$ & 0 & + & 0 & 0 & + & - & 2 & 0 & 0 & $\mathfrak{h}_{5}$ \\
$\mathfrak{h}_{6}$ & 0 & + & 1 & 0 & 0 & 0 & 2 & $*$ & $*$ & $\mathfrak{h}_{6}$ \\
$\mathfrak{h}_{6}$ & 0 & + & 1 & 0 & + & - & 2 & $*$ & $*$ & $\mathfrak{h}_{5}$ \\
$\mathfrak{h}_{7}$ & 0 & + & 2 & + & + & + & 2 & $*$ & $*$ & $\mathfrak{h}_{2}$ \\
$\mathfrak{h}_{7}$ & 0 & + & 2 & + & + & 0 & 2 & $*$ & $*$ & $\mathfrak{h}_{4}$ \\
$\mathfrak{h}_{7}$ & 0 & + & 2 & + & $*$ & - & 2 & $*$ & $*$ & $\mathfrak{h}_{5}$ \\
\hline $\mathfrak{h}_{3}$ & + & 0 & 1 & 0 & + & - & 2 & + & 0 & $\mathfrak{h}_{15}$ \\
$\mathfrak{h}_{17}$ & + & 0 & 1 & 0 & + & - & 2 & 0 & + & $\mathfrak{h}_{15}$ \\
$\mathfrak{h}_{9}$ & + & 0 & 1 & + & + & 0 & 1 & + & + & $\mathfrak{h}_{9}$ \\
$\mathfrak{h}_{9}$ & + & 0 & 2 & $*$ & 0 & - & 2 & + & + & $\mathfrak{h}_{15}$ \\
\hline $\mathfrak{h}_{6}$ & + & + & 0 & 0 & + & - & 2 & 0 & 0 & $\mathfrak{h}_{15}$ \\
$\mathfrak{h}_{4}$ & + & + & 1 & 0 & 0 & 0 & 2 & $|\rho|$ & 0 & $\mathfrak{h}_{7}, \mathfrak{h}_{16}$ \\
$\mathfrak{h}_{4}$ & + & + & 1 & 0 & + & - & 2 & + & 0 & $\mathfrak{h}_{15}$ \\
$\mathfrak{h}_{10}$ & + & + & 1 & 0 & 0 & 0 & 2 & 0 & $|\rho|$ & $\mathfrak{h}_{10}$ \\
$\mathfrak{h}_{10}$ & + & + & 1 & 0 & $*$ & - & 2 & 0 & + & $\mathfrak{h}_{15}$ \\
$\mathfrak{h}_{11}$ & + & + & 2 & + & $*$ & + & 2 & + & + & $\mathfrak{h}_{12}, \mathfrak{h}_{13}$ \\
$\mathfrak{h}_{11}$ & + & + & 2 & + & + & 0 & 2 & + & + & $\mathfrak{h}_{11}, \mathfrak{h}_{14}$ \\
$\mathfrak{h}_{11}$ & + & + & 2 & + & + & - & 2 & + & + & $\mathfrak{h}_{15}$ \\
\hline
\end{tabular}

TABLE 4.1

$\mathfrak{f}^{1}$ as a function of the parameters in the complex structure equations.

Ignoring that the same algebra $\mathfrak{f}^{1}$ occurs for distinct complex structures or different algebras, we get

TheOREm 25. Given a six-dimensional nilpotent algebra $\mathfrak{g}$, the associated Lie algebra $\mathfrak{f}^{1}(\mathfrak{g}, J)$ for all possible nilpotent complex structures $J$ are given in the rows of Table 4.2.

One observes for instance that for $\mathfrak{g}=\mathfrak{h}_{15}$ no less than seven different isomorphism classes are realized for $\mathfrak{f}^{1}(\mathfrak{g}, J)$ as $J$ runs through the space of complex structures on $\mathfrak{g}$. This is a yet another manifestation of the "jumping phenomenon" frequently seen in complex structure deformation theory.

Note that the classification of nilpotent Lie algebras in dimension 6 (see $[10,13])$ over $\mathbb{C}$ (or $\mathbb{R}$ ) has as a consequence that structure constant may be taken to always be integers, and in particular real. Thus any six-dimensional complex nilpotent algebra is self-conjugate. Then Proposition 11 implies that the complex isomorphism of Lie algebras between $\mathfrak{f}^{1}$ and $\mathfrak{h}_{n}$ generates a C-E compatible linear isomorphism $O: \mathfrak{h}_{h} \rightarrow$ $\mathfrak{h}_{n}^{*}$ such that $\operatorname{DGA}(\mathfrak{g}, J)$ and $\operatorname{DGA}\left(\mathfrak{h}_{n}, O\right)$ are isomorphic as differential Gerstenhaber algebras. In other words 
THEOREM 26. Given a six-dimensional nilpotent algebra $\mathfrak{g}$ with a nilpotent complex structure $J$, there exists a differential Gerstenhaber algebra DGA $(\mathfrak{h}, O)$ quasiisomorphic to $\mathrm{DGA}(\mathfrak{g}, J)$ if and only if the pair $(\mathfrak{g}, \mathfrak{h})$ is checked in Table 4.2.

\begin{tabular}{||l|l|l|l|l|l|l|l|l|l|l|}
\hline $\mathfrak{g} \backslash \mathfrak{f}^{1}(\mathfrak{g}, J)$ & $\mathfrak{h}_{1}$ & $\mathfrak{h}_{3}$ & $\mathfrak{h}_{4}$ & $\mathfrak{h}_{6}$ & $\mathfrak{h}_{7}$ & $\mathfrak{h}_{8}$ & $\mathfrak{h}_{9}$ & $\mathfrak{h}_{10}$ & $\mathfrak{h}_{11}$ & $\mathfrak{h}_{17}$ \\
\hline $\mathfrak{h}_{1}$ & $\checkmark$ & & & & & & & & & \\
\hline $\mathfrak{h}_{2}$ & & & & $\checkmark$ & $\checkmark$ & & & & & \\
\hline $\mathfrak{h}_{3}$ & & & & $\checkmark$ & & & & & & \\
\hline $\mathfrak{h}_{4}$ & & & & $\checkmark$ & $\checkmark$ & & & & & \\
\hline $\mathfrak{h}_{5}$ & & & & $\checkmark$ & $\checkmark$ & $\checkmark$ & & & & \\
\hline $\mathfrak{h}_{6}$ & & & & $\checkmark$ & & & & & & \\
\hline $\mathfrak{h}_{7}$ & & & $\checkmark$ & & & & & & & \\
\hline $\mathfrak{h}_{8}$ & & & & & & $\checkmark$ & & & & \\
\hline $\mathfrak{h}_{9}$ & & & & & & & $\checkmark$ & & & \\
\hline $\mathfrak{h}_{10}$ & & & & & & & & $\checkmark$ & & \\
\hline $\mathfrak{h}_{11}$ & & & & & & & & & $\checkmark$ & \\
\hline $\mathfrak{h}_{12}$ & & & & & & & & & $\checkmark$ & \\
\hline $\mathfrak{h}_{13}$ & & & & & & & & & $\checkmark$ & \\
\hline $\mathfrak{h}_{14}$ & & & & & & & & & $\checkmark$ & \\
\hline $\mathfrak{h}_{15}$ & & $\checkmark$ & $\checkmark$ & $\checkmark$ & & & $\checkmark$ & $\checkmark$ & $\checkmark$ & $\checkmark$ \\
\hline $\mathfrak{h}_{16}$ & & & $\checkmark$ & & & & & & & \\
\hline
\end{tabular}

TABLE 4.2

Isomorphism class of $\mathfrak{f}^{1}$ against underlying real algebra $\mathfrak{g}$.

The algebra $\mathfrak{h}_{3}$ appears as a candidate for $\mathfrak{f}^{1}$ in the case $\mathfrak{g}=\mathfrak{h}_{15}$. However $\mathfrak{h}_{3}$ admits no symplectic structure. This demonstrates that the differential Gerstenhaber algebra DGA $(\mathfrak{h}, O)$ does not necessarily arise from a symplectic structure, as remarked at the end of the proof of Proposition 11. The issue of whether DGA $(\mathfrak{h}, O)$ is or not coming from a symplectic structure will be deferred to future analysis.

5. Application. Once we identify the Lie algebra structure for $\mathfrak{f}^{1}(\mathfrak{g}, J)$, we have in effect identified the structure of $\operatorname{DGA}(\mathfrak{g}, J)$. Inspired by the concept of weak mirror symmetry [12], one could well look for nilpotent algebras $\mathfrak{h}$ with symplectic structure $\Omega$ whose induced differential Gerstenhaber algebra $\operatorname{DGA}(\mathfrak{h}, \Omega)$ is quasi-isomorphic to $\operatorname{DGA}(\mathfrak{g}, J)$. We shall deal with such a general question in the future. At present, we take advantage of the results in the preceding sections to address a more focused question.

Supposing that $(J, \Omega)$ is a pseudo-Kähler structure on a six-dimensional real nilpotent algebra $\mathfrak{g}$, when will there be a quasi-isomorphism

$$
\operatorname{DGA}(\mathfrak{g}, J) \rightleftharpoons \operatorname{DGA}(\mathfrak{g}, \Omega) ?
$$

Such pseudo-Kähler structures can be interpreted as weak self-mirrors, a manifestation of which - in dimension 4 - was studied in [15].

In view of Lemma 9, a quasi-isomorphism is in the present situation equivalent to an isomorphism on the degree-one level:

$$
\left(\mathfrak{f}^{1}(\mathfrak{g}, J),[-\bullet-]\right) \cong\left(\mathfrak{g}_{\mathbb{C}}^{*},[-\bullet-]_{\Omega}\right) \cong\left(\mathfrak{g}_{\mathbb{C}},[-\bullet-]\right) .
$$


Recall that a complex structure can be part of a pseudo-Kähler structure on a nilpotent algebra only if it is a nilpotent complex structure [4]. In view of Table 4.1, a solution $(\mathfrak{g}, J, \Omega)$ for the question (44) could possibly exist only if $\mathfrak{g}$ is one of the following:

$$
\mathfrak{h}_{1}, \mathfrak{h}_{6}, \mathfrak{h}_{8}, \mathfrak{h}_{9}, \mathfrak{h}_{10}, \mathfrak{h}_{11} .
$$

Below we extract from Table 4.1 the invariants for the candidate complex structures $J$ for these algebras.

\begin{tabular}{||l|c|c|c|c|c|c|c|c|c|c||}
\hline $\mathfrak{f}^{1}$ & $\epsilon$ & $|\rho|$ & $\operatorname{rank} X$ & $\left|\Delta_{1}\right|$ & $\left|\Delta_{2}\right|$ & $\left|\Delta_{1}\right|^{2}-\Delta_{2}^{2}$ & $d$ & $|B|$ & $|C|$ & $\mathfrak{g}$ \\
\hline $\mathfrak{h}_{1}$ & 0 & 0 & 0 & 0 & 0 & 0 & 0 & 0 & 0 & $\mathfrak{h}_{1}$ \\
\hline $\mathfrak{h}_{6}$ & 0 & + & 1 & 0 & 0 & 0 & 2 & $*$ & $*$ & $\mathfrak{h}_{6}$ \\
\hline $\mathfrak{h}_{8}$ & 0 & 0 & 1 & 0 & 0 & 0 & 1 & $*$ & $*$ & $\mathfrak{h}_{8}$ \\
\hline $\mathfrak{h}_{9}$ & + & 0 & 1 & + & + & 0 & 1 & + & + & $\mathfrak{h}_{9}$ \\
\hline $\mathfrak{h}_{10}$ & + & + & 1 & 0 & 0 & 0 & 2 & 0 & $|\rho|$ & $\mathfrak{h}_{10}$ \\
\hline $\mathfrak{h}_{11}$ & + & + & 2 & + & + & 0 & 2 & + & + & $\mathfrak{h}_{11}$ \\
\hline
\end{tabular}

In the next few sections, we shall take the above complex structures, and seek symplectic structures that realize the quasi-isomorphism (44). We shall analyze pseudoKähler structures on $\mathfrak{h}_{6}, \mathfrak{h}_{8}$, and $\mathfrak{h}_{11}$ in details, merely outline the discussion for $\mathfrak{h}_{9}$ and $\mathfrak{h}_{10}$, and skip the trivial case $\mathfrak{h}_{1}$ completely.

5.1. $\mathfrak{h}_{6}$. Given the invariants, the reduced structure equations (25) are

$$
d \omega^{1}=0, \quad d \omega^{2}=0, \quad d \omega^{3}=\omega^{1} \omega^{2}+A \omega^{1} \bar{\omega}^{1}+B \omega^{1} \bar{\omega}^{2}+C \omega^{2} \bar{\omega}^{1}+D \omega^{2} \bar{\omega}^{2} .
$$

Since $\Delta_{1}=0$ there exists a constant $\lambda$ such that either

$$
d \omega^{3}=\omega^{1} \omega^{2}+\left(\omega^{1}+\lambda \omega^{2}\right)\left(A \bar{\omega}^{1}+B \bar{\omega}^{2}\right) \quad \text { or } \quad d \omega^{3}=\omega^{1} \omega^{2}+\left(\lambda \omega^{1}+\omega^{2}\right)\left(C \bar{\omega}^{1}+D \bar{\omega}^{2}\right) .
$$

The condition $\Delta_{2}=0$ implies that in either case, there exists a change of complex basis so that the structure equations transform to

$$
d \omega^{1}=0, \quad d \omega^{2}=0, \quad d \omega^{3}=\omega^{1} \omega^{2}+\omega^{1} \bar{\omega}^{2} .
$$

It follows that the structure equations for $\left(\mathfrak{f}^{1},[-\bullet-], \bar{\partial}\right)$ are

$$
\left[T_{1}, T_{2}\right]=-T_{3}, \quad\left[T_{2}, \bar{\omega}^{3}\right]=-\bar{\omega}^{1}, \quad \bar{\partial} T_{1}=\bar{\omega}^{2} \wedge T_{3}, \quad \bar{\partial} \bar{\omega}^{3}=\bar{\omega}^{1} \wedge \bar{\omega}^{2} .
$$

Due to [6, Lemma 3.4], given the complex structure equations, any (1,1)-form of a compatible symplectic structure is given by

$$
\Omega=a_{1} \omega^{1} \bar{\omega}^{1}+b_{2} \omega^{2} \bar{\omega}^{2}+\bar{a}_{2} \bar{\omega}^{1} \omega^{2}+a_{2} \omega^{1} \bar{\omega}^{2}+a_{3}\left(\omega^{1} \bar{\omega}^{3}+\bar{\omega}^{1} \omega^{3}\right),
$$

where $a_{1}$ and $b_{2}$ are imaginary numbers and $a_{3}$ is a real number. This 2 -form is non-degenerate if and only if $b_{2} \neq 0$ and $a_{3} \neq 0$.

Setting $\omega^{1}=e^{2}+i e^{3}, \omega^{2}=-\frac{1}{2}\left(e^{1}+i e^{4}\right)$ and $\omega^{3}=e^{5}+i e^{6}$, reduces the complex structure equation to

$$
d e^{5}=e^{12}, \quad d e^{6}=e^{13} .
$$

Set $a_{1}=\frac{i}{2} a, b_{2}=2 i b, a_{2}=c+i k$ and $a_{3}=\ell / 2$ with $b \neq 0$ and $\ell \neq 0$. Then the symplectic structure is

$$
\Omega=a e^{23}+b e^{14}+c\left(e^{12}-e^{34}\right)-k\left(e^{13}+e^{24}\right)+\ell\left(e^{25}+e^{36}\right) .
$$


Using the contraction with $\Omega$ as an isomorphism from $\mathfrak{h}_{6}$ and $\mathfrak{h}_{6}^{*}$, we obtain a Lie bracket on $\mathfrak{h}_{6}^{*}$ such that

$$
b\left[e^{4}, e^{5}\right]_{\Omega}=e^{2}, \quad b\left[e^{4}, e^{6}\right]_{\Omega}=e^{3}, \quad b l\left[e^{5}, e^{6}\right]_{\Omega}=\left(c e^{2}-k e^{3}\right) .
$$

It is now apparent that the linear map

$$
T_{1} \mapsto e^{5}+\frac{k}{\ell} e^{4}, \quad T_{2} \mapsto b e^{4}, \quad T_{3} \mapsto e^{2}, \quad \bar{\omega}^{1} \mapsto-e^{3}, \quad \bar{\omega}^{2} \mapsto e^{1}, \quad \bar{\omega}^{3} \mapsto e^{6}+\frac{c}{\ell} e^{4} .
$$

yields an isomorphism of differential Gerstenhaber algebras.

Note that the isomorphism exists so long as the symplectic form $\Omega$ and the designated complex structure $J$ together form a pseudo-Kähler structure.

Proposition 27. Let $J$ be any integrable complex structure on $\mathfrak{h}_{6}$. Let $\Omega$ be any symplectic form on $\mathfrak{h}_{6}$ of type $(1,1)$ with respect to $J$. Then the differential Gerstenhaber algebras $\mathrm{DGA}\left(\mathfrak{h}_{6}, J\right)$ and $\mathrm{DGA}\left(\mathfrak{h}_{6}, \Omega\right)$ are isomorphic.

5.2. $\mathfrak{h}_{8}$. In this case, the invariants yield the following structure equations.

$$
d \omega^{1}=0, \quad d \omega^{2}=0, \quad d \omega^{3}=A \omega^{1} \bar{\omega}^{1}+B \omega^{1} \bar{\omega}^{2}+C \omega^{2} \bar{\omega}^{1}+D \omega^{2} \bar{\omega}^{2},
$$

where the arrays $(A, B)$ and $(C, D)$ are linearly dependent but are not identically zero. After a change of complex coordinates, they could be reduced to

$$
d \omega^{1}=0, \quad d \omega^{2}=0, \quad d \omega^{3}=\omega^{1} \bar{\omega}^{1} .
$$

The induced structure equations for $\mathfrak{f}^{1}$ are

$$
\left[T_{1}, \bar{\omega}^{3}\right]=-\bar{\omega}^{1}, \quad \bar{\partial} T_{1}=\bar{\omega}^{1} \wedge T_{3} .
$$

By choosing

$$
\omega^{1}=e^{1}+i e^{2}, \quad \omega^{2}=e^{3}+i e^{4}, \quad \omega^{3}=-2\left(e^{5}+i e^{6}\right),
$$

then the real structure equation is indeed the standard one for $\mathfrak{h}_{8}$ :

$$
d e^{6}=e^{1} \wedge e^{2} .
$$

Again, due to [6, Lemma 3.4] given the complex structure equations, any symplectic $(1,1)$-form is given by

$$
\Omega=a e^{12}+b e^{34}+x\left(e^{13}+e^{24}\right)-y\left(e^{23}-e^{14}\right)-u\left(e^{15}+e^{26}\right)+v\left(e^{25}-e^{16}\right),
$$

where $a, b, x, y, u, v$ are real numbers. $\Omega$ is non-degenerate when $b \neq 0$ and $u^{2}+v^{2} \neq 0$. Then the induced Lie bracket on $\mathfrak{h}_{8}^{*}$ is given by

$$
\left[-u e^{5}-v e^{6} \cdot v e^{5}-u e^{6}\right]_{\Omega}=-\left(u e^{2}+v e^{1}\right) .
$$

Since $u^{2}+v^{2} \neq 0$, it is an elementary exercise to find an isomorphism from $\operatorname{DGA}\left(\mathfrak{h}_{8}, J\right)$ to $\operatorname{DGA}\left(\mathfrak{h}_{8}, \Omega\right)$. For instance, when $v \neq 0$, one could construct an isomorphism so that

$$
T_{1} \mapsto-u e^{5}-v e^{6}, \quad \bar{\omega}^{3} \mapsto v e^{5}-u e^{6}, \quad \bar{\omega}^{1} \mapsto u e^{2}+v e^{1} .
$$

As in the last section, the computation demonstrates more than simply the existence of a self-mirror pair of complex and symplectic structure.

Proposition 28. Let $J$ be any integrable complex structure on $\mathfrak{h}_{8}$. Let $\Omega$ be any symplectic form on $\mathfrak{h}_{8}$ of type $(1,1)$ with respect to $J$. Then the differential Gerstenhaber algebras $\mathrm{DGA}\left(\mathfrak{h}_{8}, J\right)$ and $\mathrm{DGA}\left(\mathfrak{h}_{8}, \Omega\right)$ are isomorphic. 
5.3. $\mathfrak{h}_{9}$. The complex structure equations are given by

$$
d \omega^{1}=0, \quad d \omega^{2}=\omega^{1} \bar{\omega}^{1}, \quad d \omega^{3}=B \omega^{1} \bar{\omega}^{2}+C \omega^{2} \bar{\omega}^{1},
$$

where $B \neq 0$ and $C \neq 0$. Therefore, we can normalize to

$$
d \omega^{1}=0, \quad d \omega^{2}=-\frac{1}{2} \omega^{1} \bar{\omega}^{1}, \quad d \omega^{3}=\frac{1}{2} \omega^{1} \bar{\omega}^{2}+\frac{1}{2} \omega^{2} \bar{\omega}^{1} .
$$

Choose

$$
\omega^{1}=e^{1}+i e^{2}, \quad \omega^{2}=e^{4}+i e^{5}, \quad \omega^{3}=e^{6}+i e^{3},
$$

to the effect that $\Omega=e^{13}-e^{26}-e^{45}$ is a pseudo-Kähler form so that $\mathfrak{f}^{1}\left(\mathfrak{h}_{9}, J\right)$ is isomorphic to $\left(\mathfrak{h}_{9}^{*},[-\bullet-]_{\Omega}\right)$.

5.4. $\mathfrak{h}_{10}$. The complex structure equation is given by

$$
d \omega^{1}=0, \quad d \omega^{2}=\omega^{1} \bar{\omega}^{1}, \quad d \omega^{3}=\omega^{1} \omega^{2}+\omega^{2} \bar{\omega}^{1}
$$

In this case, when we choose

$$
\omega^{1}=e^{1}+i e^{2}, \quad \omega^{2}=e^{3}+i e^{4}, \quad \omega^{3}=e^{5}+i e^{6},
$$

then $\Omega=i\left(e^{16}-e^{25}-e^{34}\right)$ is a pseudo-Kähler form such that $\mathfrak{f}^{1}\left(\mathfrak{h}_{10}, J\right)$ is isomorphic to $\left(\mathfrak{h}_{10}^{*},[-\bullet-]_{\Omega}\right)$.

5.5. $\mathfrak{h}_{11}$. This case requires a careful analysis. We show that for every pseudo-Kähler pair $(J, \Omega)$ on $\mathfrak{h}_{11}$ the differential Gerstenhaber algebras DGA $\left(\mathfrak{h}_{11}, J\right)$ and $\operatorname{DGA}\left(\mathfrak{h}_{11}, \Omega\right)$ are non-isomorphic. To this end we shall suppose that $\Phi: \operatorname{DGA}\left(\mathfrak{h}_{11}, J\right) \rightarrow \operatorname{DGA}\left(\mathfrak{h}_{11}, \Omega\right)$ is a quasi-isomorphism of differential Gerstenhaber algebras obtained from a pseudo-Kähler pair $(J, \Omega)$ and establish a contradiction.

Note that $\mathfrak{h}_{11}$ is distinguished by the data: $n=(3,5)$ and $\left|\Delta_{1}\right|^{2}=\Delta_{2}^{2}>0$ for any $J$, see Lemma 13 and Lemma 19. Furthermore, for any complex structure on $\mathfrak{h}_{11}$ we may always choose a basis of $(1,0)$-forms such that

$$
d \omega^{1}=0, \quad d \omega^{2}=\omega^{1} \bar{\omega}^{1}, \quad d \omega^{3}=\omega^{1} \omega^{2}+B \omega^{1} \bar{\omega}^{2}+C \omega^{2} \bar{\omega}^{1}
$$

Choosing $\omega$ this way, the constraints $n_{2}=5$ and $\left|\Delta_{1}\right|^{2}=\Delta_{2}^{2}>0$ on the invariants are equivalent to $B$ being real, $|C|^{2}=(B-1)^{2}$ and $B C \neq 0$. We shall use this extensively below. Precisely these conditions on $B$ and $C$ give

$$
d\left((B-1) \omega^{3}+C \bar{\omega}^{3}\right)=\left((B-1) \omega^{1}+C \bar{\omega}^{1}\right)\left(\omega^{2}+\bar{\omega}^{2}\right)
$$

whence

$$
V_{1}\left(\mathfrak{h}_{11}\right)=\left\langle\omega^{1}, \bar{\omega}^{1}, \omega^{2}+\bar{\omega}^{2}\right\rangle, \quad V_{2}\left(\mathfrak{h}_{11}\right)=\left\langle\omega^{1}, \bar{\omega}^{1}, \omega^{2}, \bar{\omega}^{2},(B-1) \omega^{3}+C \bar{\omega}^{3}\right\rangle
$$

Solving the equations $d \Omega=0$ and $\Omega=\bar{\Omega}$ in the space of $(1,1)$-forms gives

$$
\Omega=a_{1} \omega^{1} \bar{\omega}^{1}+a_{3}(B+1) \omega^{2} \bar{\omega}^{2}+a_{2} \omega^{1} \bar{\omega}^{2}-\bar{a}_{2} \omega^{2} \bar{\omega}^{1}+a_{3}\left(\omega^{1} \bar{\omega}^{3}+\omega^{3} \bar{\omega}^{1}\right),
$$

where $a_{1}+\bar{a}_{1}=0=a_{3}+\bar{a}_{3}$ and $a_{1} a_{3}(B+1) \neq 0$ if and only if $\Omega$ is non-degenerate ${ }^{1}$. Therefore $\Omega\left(T_{1}\right)=a_{1} \bar{\omega}^{1}+a_{2} \bar{\omega}^{2}+a_{3} \bar{\omega}^{3}, \Omega\left(T_{2}\right)=-\bar{a}_{2} \bar{\omega}^{1}+a_{3}(B+1) \bar{\omega}^{2}, \Omega\left(T_{3}\right)=a_{3} \bar{\omega}^{1}$

${ }^{1}$ This also means: nilpotent complex structures on $\mathfrak{h}_{11}$ with $B=-1$ have no compatible symplectic forms. 
and

$$
\begin{gathered}
\omega^{1}=-\frac{1}{a_{3}} \Omega\left(\bar{T}_{3}\right), \quad \omega^{2}=-\frac{1}{(B+1) a_{3}} \Omega\left(\bar{T}_{2}-\frac{a_{2}}{a_{3}} \bar{T}_{3}\right), \\
\omega^{3}=-\frac{1}{a_{3}} \Omega\left(\bar{T}_{1}+\frac{\bar{a}_{2}}{(B+1) a_{3}} \bar{T}_{2}-\frac{(B+1) a_{1} a_{3}+\left|a_{2}\right|^{2}}{(B+1) a_{3}^{2}} \bar{T}_{3}\right) .
\end{gathered}
$$

Now the brackets are easily computed

$$
\begin{gathered}
{\left[\omega^{2} \bullet \omega^{3}\right]_{\Omega}=-\frac{1}{(B+1) a_{3}} \omega^{1}, \quad\left[\omega^{2} \bullet \bar{\omega}^{3}\right]_{\Omega}=-\frac{1}{(B+1) a_{3}}\left(\bar{C} \omega^{1}+B \bar{\omega}^{1}\right),} \\
{\left[\omega^{3} \bullet \bar{\omega}^{3}\right]_{\Omega}=-\frac{1}{(B+1) a_{3}^{2}}\left(\left(a_{2}+\bar{a}_{2} \bar{C}\right) \omega^{1}-\left(\bar{a}_{2}+a_{2} C\right) \bar{\omega}^{1}\right)-\frac{B+1}{a_{3}}\left(\omega^{2}+\bar{\omega}^{2}\right),}
\end{gathered}
$$

and the lower central series for $\left(\mathfrak{h}_{11}^{*},[\cdot \bullet \cdot]_{\Omega}\right)$ is

$$
\left(\mathfrak{h}_{11}^{*}\right)_{1}=\left\langle\omega^{1}, \bar{\omega}^{1}, \omega^{2}+\bar{\omega}^{2}\right\rangle, \quad\left(\mathfrak{h}_{11}^{*}\right)_{2}=\left\langle(B-1) \omega^{1}+C \bar{\omega}^{1}\right\rangle, \quad\left(\mathfrak{h}_{11}^{*}\right)_{3}=\{0\},
$$

while the ascending series is

$$
D^{1}\left(\mathfrak{h}_{11}^{*}\right)=\left\langle\omega^{1}, \bar{\omega}^{1}\right\rangle, \quad D^{2}\left(\mathfrak{h}_{11}^{*}\right)=\left\langle\omega^{1}, \bar{\omega}^{1}, \omega^{2}, \bar{\omega}^{2}\right\rangle, \quad D^{3}\left(\mathfrak{h}_{11}^{*}\right)=\mathfrak{h}_{11}^{*} .
$$

On the other hand, the structure equations for $\operatorname{DGA}\left(\mathfrak{h}_{11}, J\right)$ given by $(61)$ are

$$
\begin{gathered}
\bar{\partial} T_{1}=\bar{\omega}^{1} \wedge T_{2}+B \bar{\omega}^{2} \wedge T_{3}, \quad \bar{\partial} T_{2}=C \bar{\omega}^{1} \wedge T_{3}, \quad \bar{\partial} \bar{\omega}^{3}=\bar{\omega}^{1} \wedge \bar{\omega}^{2}, \\
{\left[T_{1} \bullet T_{2}\right]=-T_{3}, \quad\left[T_{1} \bullet \bar{\omega}^{2}\right]=-\bar{\omega}^{1}, \quad\left[T_{1} \bullet \bar{\omega}^{3}\right]=-\bar{C} \bar{\omega}^{2}, \quad\left[T_{2} \bullet \bar{\omega}^{3}\right]=-B \bar{\omega}^{1} .}
\end{gathered}
$$

Writing $\mathfrak{f}^{1}$ for the space of degree one elements in $\operatorname{DGA}\left(\mathfrak{h}_{11}, J\right)$ we have

$$
\begin{gathered}
V_{1}\left(\mathfrak{f}^{1}\right)=\left\langle T_{3}, \bar{\omega}^{1}, \bar{\omega}^{2}\right\rangle, \quad V_{2}\left(\mathfrak{f}^{1}\right)=\left\langle T_{2}, T_{3}, \bar{\omega}^{1}, \bar{\omega}^{2}, \bar{\omega}^{3}\right\rangle, \\
\left(\mathfrak{f}^{1}\right)_{1}=\left\langle T_{3}, \bar{\omega}^{1}, \bar{\omega}^{2}\right\rangle, \quad\left(\mathfrak{f}^{1}\right)_{2}=\left\langle\bar{\omega}^{1}\right\rangle, \quad\left(\mathfrak{f}^{1}\right)_{3}=\{0\}, \\
D^{1}\left(\mathfrak{f}^{1}\right)=\left\langle\bar{\omega}^{1}, T_{3}\right\rangle, \quad D^{2}\left(\mathfrak{f}^{1}\right)=\left\langle\bar{\omega}^{1}, T_{3}, \bar{\omega}^{2}, T_{2}\right\rangle, \quad D^{3}\left(\mathfrak{f}^{1}\right)=\mathfrak{f}^{1} .
\end{gathered}
$$

By Proposition 10, any quasi-isomorphism $\Phi: \operatorname{DGA}\left(\mathfrak{h}_{11}, J\right) \rightarrow \operatorname{DGA}\left(\mathfrak{h}_{11}, \Omega\right)$ must be an isomorphism of DGAs and therefore maps $V_{k}\left(\mathfrak{f}^{1}\right)$ isomorphically onto $V_{k}\left(\mathfrak{h}_{11}\right)$, $\left(\mathfrak{f}^{1}\right)_{j}$ isomorphically onto $\left(\mathfrak{h}_{11}^{*}\right)_{j}$ and similarly for the ascending sequences. It follows that complex constants $\phi_{n}^{m}$ exist such that

$$
\begin{aligned}
& \Phi\left(\bar{\omega}^{1}\right)=\phi_{1}^{1}\left((B-1) \omega^{1}+C \bar{\omega}^{1}\right), \\
& \Phi\left(T_{3}\right)=\phi_{1}^{2} \omega^{1}+\phi_{2}^{2} \bar{\omega}^{1} \\
& \Phi\left(\bar{\omega}^{2}\right)=\phi_{1}^{3} \omega^{1}+\phi_{2}^{3} \bar{\omega}^{1}+\phi_{3}^{3}\left(\omega^{2}+\bar{\omega}^{2}\right), \\
& \Phi\left(T_{2}\right)=\phi_{1}^{4} \omega^{1}+\phi_{2}^{4} \bar{\omega}^{1}+\phi_{3}^{4} \omega^{2}+\phi_{4}^{4} \bar{\omega}^{2}, \\
& \Phi\left(\bar{\omega}^{3}\right)=\phi_{1}^{5} \omega^{1}+\phi_{2}^{5} \bar{\omega}^{1}+\phi_{3}^{5} \omega^{2}+\phi_{4}^{5} \bar{\omega}^{2}+\phi_{5}^{5}\left((B-1) \omega^{3}+C \bar{\omega}^{3}\right), \\
& \Phi\left(T_{1}\right)=\phi_{1}^{6} \omega^{1}+\phi_{2}^{6} \bar{\omega}^{1}+\phi_{3}^{6} \omega^{2}+\phi_{4}^{6} \bar{\omega}^{2}+\phi_{5}^{6} \omega^{3}+\phi_{6}^{6} \bar{\omega}^{3} .
\end{aligned}
$$

More detailed information is now obtained by applying $\Phi$ to the structure equations. From $d\left(\Phi\left(T_{2}\right)\right)=C \Phi\left(\bar{\omega}^{1}\right) \wedge \Phi\left(T_{3}\right)$ we get

$$
\left(\phi_{3}^{4}-\phi_{4}^{4}\right)=C \phi_{1}^{1}\left((B-1) \phi_{2}^{2}-C \phi_{1}^{2}\right) .
$$


The $\left((B-1) \omega^{1}+C \bar{\omega}^{1}\right)\left(\omega^{2}+\bar{\omega}^{2}\right)$-component of $d\left(\Phi\left(\bar{\omega}^{3}\right)\right)=\Phi\left(\bar{\omega}^{1}\right) \wedge \Phi\left(\bar{\omega}^{3}\right)$ gives

$$
\phi_{5}^{5}=\phi_{1}^{1} \phi_{3}^{3} .
$$

Eliminating $\phi_{5}^{6}$ and $\phi_{6}^{6}$ in the equations derived from $d\left(\Phi\left(T_{1}\right)\right)=\Phi\left(\bar{\omega}^{1}\right) \wedge \Phi\left(T_{2}\right)+$ $B \Phi\left(\bar{\omega}^{2}\right) \wedge \Phi\left(T_{3}\right)$ leads to $C \phi_{1}^{1}\left(\phi_{3}^{4}-\phi_{4}^{4}\right)+\phi_{3}^{3}\left((B-1) \phi_{2}^{2}-C \phi_{1}^{2}\right)=0$. The result of inserting (65) in this is $\left(\left(C \phi_{1}^{1}\right)^{2}+\phi_{3}^{3}\right)\left((B-1) \phi_{2}^{2}-C \phi_{1}^{2}\right)=0$. Since $\Phi$ is a linear isomorphism $\Phi\left(\bar{\omega}^{1}\right)$ and $\Phi\left(T_{3}\right)$ are linearly independent, and so

$$
\phi_{3}^{3}=-\left(C \phi_{1}^{1}\right)^{2} .
$$

The equation $\left[\Phi\left(T_{1}\right) \bullet \Phi\left(\bar{\omega}^{2}\right)\right]_{\Omega}=-\Phi\left(\bar{\omega}^{1}\right)$ is equivalent to

$$
C(B+1) a_{3} \phi_{1}^{1}=\phi_{3}^{3}\left(C \phi_{5}^{6}-(B-1) \phi_{6}^{6}\right)
$$

while the $\omega^{2}+\bar{\omega}^{2}$-component of $\left[\Phi\left(T_{1}\right) \bullet \Phi\left(\bar{\omega}^{3}\right)\right]_{\Omega}=-\bar{C} \Phi\left(\bar{\omega}^{2}\right)$ gives

$$
a_{3} \bar{C} \phi_{3}^{3}=(B+1) \phi_{5}^{5}\left(C \phi_{5}^{6}-(B-1) \phi_{6}^{6}\right) .
$$

Substituting first (66), and then equations (68) and (67) in (69) yields

$$
a_{3}|C|^{2} C\left(\phi_{1}^{1}\right)^{2}=-a_{3}(B+1)^{2} C\left(\phi_{1}^{1}\right)^{2} .
$$

Since $|C|^{2}=(B-1)^{2}$, this implies $a_{3} C \phi_{1}^{1}=0$ and so establishes our contradiction: if $a_{3}=0$ then $\Omega$ is degenerate, $C=0$ cannot be realized on $\mathfrak{h}_{11}$, and if $\phi_{1}^{1}=0$ then $\Phi$ is no isomorphism.

5.6. Conclusion. The computation in the past few paragraphs is summarized in the following observation.

TheOREM 29. A six-dimensional nilpotent algebra $\mathfrak{g}$ admits a pseudo-Kähler structure $(J, \Omega)$ such that $\operatorname{DGA}(\mathfrak{g}, J)$ is quasi-isomorphic to $\operatorname{DGA}(\mathfrak{g}, \Omega)$ if and only if $\mathfrak{g}$ is one of $\mathfrak{h}_{1}, \mathfrak{h}_{6}, \mathfrak{h}_{8}, \mathfrak{h}_{9}$ and $\mathfrak{h}_{10}$.

REMARK 2. In this paper, we have dealt exclusively with Lie algebras. However, it is possible to extend the whole discussion to nilmanifolds $M=G / \Gamma$, i.e. quotients of simply connected nilpotent Lie groups $G$ with respect to co-compact lattices $\Gamma$. Indeed, the de Rham cohomology of $M$ is given by invariant forms on $G$ [14]. Therefore, when $M$ has an invariant symplectic structure, the invariant differential Gerstenhaber algebra $\operatorname{DGA}(\mathfrak{g}, \Omega)$ provides a minimal model for the differential Gerstenhaber algebra over the space of sections of the exterior differential forms on the nilmanifold $M$.

Similarly, for nilpotent complex structures on nilmanifolds there are partial results proving that the space of invariant sections is a minimal model of the Dolbeault cohomology with coefficients in the holomorphic tangent sheaf [2] [3] [4]. Given such a result for a particular class of complex structures (e.g. abelian complex structure [3]), Theorem 29 can be paraphrased as a statement about quasi-isomorphisms of DGAs over nilmanifolds with pseudo-Kähler structures.

Acknowledgments. We are grateful to S. Chiossi for reading the manuscript and for his extremely useful comments.

R. Cleyton is partially supported by the University of California at Riverside, the Junior Research Group "Special Geometries in Mathematical Physics" of the Volkswagen foundation and the SFB 647 "Space-Time-Matter", of the DFG. 


\section{REFERENCES}

[1] M. L. Barberis, I. G. Dotti \& R. J. Miatello, On certain locally homogeneous Clifford manifolds, Ann. Glob. Anal. Geom., 13 (1995), pp. 513-518.

[2] S. Console \& A. Fino, Dolbeault cohomology of compact nilmanifolds, Transform. Groups., 6 (2001), pp. 111-124.

[3] S. Console, A. Fino \& Y. S. Poon, Stability of abelian complex structures, Internat. J. Math., 17 (2006), pp. 401-416.

[4] L. A. Cordero, M. Fernández, A. Gray \& L. Ugarte, Nilpotent complex structures on compact nilmanifolds, Rend. Circolo Mat. Palermo, 49 suppl. (1997), pp. 83-100.

[5] L. A. Cordero, M. Fernández, A. Gray \& L. Ugarte, Compact nilmanifolds with nilpotent complex structures: Dolbeault cohomology, Trans. Amer. Math. Soc., 352 (2000), pp. 54055433.

[6] L. A. Cordero, M. Fernández \& L. Ugarte, Pseudo-Kähler metrics on six-dimensional nilpotent Lie algebras, J. Geom. Phys., 50 (2004), pp. 115-137.

[7] M. Gerstenhaber, The cohomology structure on an associative ring, Ann. Math., 78 (1963), pp. 267-288.

[8] W. M. Goldman \& J. J. Millson, The homotopy invariance of the Kuranishi space, Illinois J. Math., 34 (1990), pp. 397-368.

[9] K. C. H. Mackenzie, General Theory of Lie Groupoids and Lie Algebroids, London Math. Soc. Lecture Notes Series, 213 (2005).

[10] L. Magnin, Sur les algèbres de Lie nilpotentes de dimension $\leq$ 7, J. Geom. Phys., 3, (1986), pp. 119-144.

[11] S. A. Merkulov, Frobenius $\infty$ invariants of homotopy Gerstenhaber algebras, I, Duke Math. J., 105 (2000), pp. 411-461.

[12] S. A. Merkulov, A note on extended complex manifolds. Symplectic and contact topology: interactions and perspectives (Toronto, ON/Montreal, QC, 2001), pp. 145-155, Fields Inst. Commun., 35, Amer. Math. Soc., Providence, RI, 2003.

[13] V. V. Morozov, Classification of nilpotent Lie algebras of sixth order, Izv. Vysš. Učebn. Zaved. Matematika, 4 (1958), pp. 161-171.

[14] K. Nomizu, On the cohomology of compact homogenous spaces of nilpotent Lie groups, Ann. Math., 59 (1954), pp. 531-538.

[15] Y. S. Poon, Extended deformation of Kodaira surfaces, J. reine angew. Math., 590 (2006), pp. $45-65$.

[16] S. Salamon, Complex structures on nilpotent Lie algebras, J. Pure Appl. Algebra, 157 (2001), pp. 311-333.

[17] L. Ugarte, Hermitian structures on six dimensional nilmanifolds, Transformation Groups, 12 (2007), pp. 175-202.

[18] J. ZHOU, Homological perturbation theory and mirror symmetry, Acta Math. Sinica, English Version., 19 (2003), pp. 695-714. 
